This item was submitted to Loughborough's Research Repository by the author.

Items in Figshare are protected by copyright, with all rights reserved, unless otherwise indicated.

\title{
An improved measure of deaths due to COVID-19 in England and Wales
}

PLEASE CITE THE PUBLISHED VERSION

https://dx.doi.org/10.2139/ssrn.3635548

PUBLISHER

SSRN

VERSION

AO (Author's Original)

LICENCE

CC BY-NC-ND 4.0

REPOSITORY RECORD

Sam, Williams, Crookes Alasdair, Karligash Glass, and Anthony Glass. 2020. "An Improved Measure of Deaths Due to COVID-19 in England and Wales”. Loughborough University.

https://hdl.handle.net/2134/12579629.v1. 


\title{
An Improved Measure of Deaths due to COVID-19 in England and Wales
}

\author{
Sam Williams*, Alasdair Crookes ${ }^{\#}$, Karli Glass ${ }^{\S}$ and Anthony Glass ${ }^{\circledR}$
}

25 June 2020

\begin{abstract}
'How many deaths in England and Wales are due to COVID-19?' An 'excess deaths' measure of COVID deaths is preferable, but a limitation of the current metric (a comparison to a 5 year average for the same week), is that it attributes all the variation in mortality to COVID-19. This likely means the metric is overstated because there are other drivers of mortality. We account for other drivers including the lockdown using novel Poisson models for deaths (in totality; by age category; for males; and females). Novel COVID identifier variables (a variation on a dummy variable) are used to estimate weekly deviations in COVID deaths about the mean weekly estimate. For 17 or 24 April 2020 - 8 May 2020, we predict that total weekly COVID deaths are considerably below the 5 year average excess deaths - on average per week, 4670 or 4727 deaths lower $(54 \%$ or $63 \%$ lower, respectively).
\end{abstract}

JEL Classification: C54; I18

Key words: Overreporting of COVID deaths; Lockdown policy; Mean COVID deaths estimate; Deviations from the mean estimate; Demographics

\footnotetext{
* Economic Insight, 125 Old Broad Street, London, EC2N 1AR. Email: sam.williams@economic-insight.com. The authors acknowledge comments on an earlier version of this paper from colleagues at Economic Insight.

\# Economic Insight, 125 Old Broad Street, London, EC2N 1AR. Email: alasdair.crookes@economic-insight.com

$\S$ Corresponding author. School of Business and Economics, Loughborough University, Leicestershire, LE11 3TU, UK.Email: k.glass@lboro.ac.uk

" Sheffield University Management School, The University of Sheffield, Conduit Road, Sheffield, S10 1FL, UK. Email: a.j.glass@sheffield.ac.uk
} 


\section{Introduction}

There is no doubt that there has been a substantial number of deaths in the world due to COVID19. However, coupled with this is a great deal of uncertainty about the precise number of deaths that can be attributed to COVID. This is a problem across countries and relates to the limitations of current measures of COVID deaths. To fully understand the effect of the pandemic, an approach that accurately quantifies deaths in countries due to COVID is needed. In this paper we set out such an approach for a single country that, in due course, can be applied more widely by addressing the question of: how many deaths in England and Wales are due to COVID-19?

At present there are two approaches to measuring COVID deaths in England and Wales. The first is referred to as 'COVID associated deaths', and the second is referred to as 'excess deaths'. The method for recording COVID associated deaths has varied over time. Up until April $29^{\text {th }} 2020$, it reflected the number of people who had died in National Health Service (NHS)-commissioned services who had also tested positive for COVID-19. After April 29 $9^{\text {th }}$, NHS Trusts recorded deaths using the 'COVID-19 Patient Notification System'. Under this system, deaths can be recorded as being due to COVID even in the absence of a positive test (i.e., where a test has not occurred, or sometimes even where a prior test was negative). In addition, local health teams notify Public Health England (PHE) of suspected COVID deaths (primarily outside of a hospital setting). A third channel for recording COVID associated deaths is also used. Namely, positive COVID test records submitted by laboratories and identified via the Second Generation Surveillance System (SGSS) are cross checked against the NHS central register of patients. If this shows a patient with a positive test has subsequently died, the death is classified as 'COVID associated'. ${ }^{1}$ Alternatively, the 'excess deaths' approach to measuring COVID mortality compares the total number of deaths in any given week (all-cause mortality) with the equivalent figure in the same week averaged over the prior 5 years. The data for this measure is entirely sourced from the Office of National Statistics (ONS) and is based on total death registrations. ${ }^{2}$

To differing degrees, both measures of COVID deaths in England and Wales are inaccurate. At this juncture we only summarise the reasons for these inaccuracies, because in the next section we revisit these limitations in detail. In short, the COVID associated death method is particularly problematic, because there is no reliable record of causality between COVID infection and the subsequent death. This limitation is especially important if one were seeking to compare COVID deaths across countries. That is to say, not only will no one method of recording be 'perfect', but each individual country may have used different rules for determining 'when' to record COVID on a death certificate.

\footnotetext{
${ }^{1}$ This information was published by HM Government (2020).

${ }^{2}$ As described further by the ONS (2020).
} 
In light of the above measurement problem with COVID associated deaths, a consensus has formed around excess deaths as being a preferred measurement approach. To illustrate, Professor Chris Whitty, the Chief Medical Officer for the UK, has advocated this, explaining as follows: "the metric we should be using... is all-cause mortality adjusted for age. That is the key metric. We've discussed it today amongst a lot of the scientists. Everybody agrees this is the key metric and the reason for that is every country measures its COVID cases in a slightly different way. "3 Similarly EuroMOMO (European Mortality Monitoring) (2020) has elected to monitor COVID using the excess deaths (all mortalities) approach, explaining that: "there is a risk of countries sharing incompatible information if different methodologies are used." The Health Foundation (2020) has stated that: "excess deaths is a better measure than COVID-19 deaths of the pandemic's total mortality [because it] does not depend on how COVID-19 deaths are recorded."

We are also in agreement that because of the above measurement problem with COVID associated deaths, excess deaths is a superior approach to understanding the true number of deaths due to COVID. However, there are two limitations with the excess deaths method, or at least, the current 'simple' application of it in the context of the COVID-19 pandemic (whereby contemporaneous weekly deaths are typically compared to the 5 year average for the same week).

The first limitation of the excess deaths method arises because COVID-19 deaths are highly concentrated in the elderly. This may mean that a proportion of the deaths reported to date would have occurred at some future point in the year. Thus, rather than these deaths being truly 'excessive' due to COVID, they might instead have been modestly 'brought forward' (the implication being that, for example, deaths due to influenza in the elderly may be lower in winter 2020 than they otherwise would have been). This limitation in the excess deaths method cannot be fully overcome until a full years' worth of data is available. Until that point, caution must therefore be attached to any excess deaths measure. What one might say is that, because it would seem unlikely that at least some deaths have not been brought forward from later in 2020 (rather than being incremental), this most likely means there will be some overstatement of excess deaths. One cannot, however, say by 'how much'.

The second limitation of the excess deaths method is that there are many drivers of all-cause mortalities. Therefore, an approach that attributes all of the difference between current weekly death numbers and the 5 year average to COVID omits important information (and, crucially, a distinction between COVID 19 itself and any Government policy response; the latter of which may affect mortality both positively and negatively). This, too, will likely lead to some overstatement of excess deaths. Given the excess deaths approach is superior and sufficient time has not elapsed

\footnotetext{
${ }^{3}$ From Boris Johnson's UK Coronavirus Briefing Transcript, April 30 2020.
} 
to enable us to fully overcome its first limitation, the primary focus of this paper is on overcoming the second limitation of the approach. We do so by applying an excess deaths framework more robustly, which involves controlling for factors other than COVID that might be causing variations in weekly deaths in England and Wales. Our hope is that by robustly identifying factors that impact the death rate in England and Wales, we can help further thinking as to the appropriate methods for estimating and comparing excess COVID deaths across countries in due course; and also further the understanding of the impact of countries' respective policy responses.

To model mortality, a Poisson model is frequently estimated (e.g., Michener and Tighe, 1992, Coelho and Nunes, 2011, Sekhri and Storeygard, 2014, and Regidor et al., 2016, to name but a few studies). Such an approach is well-suited to this task, as it is a count data method and assuming that mortality is Poisson distributed corresponds to the mutually exclusive outcomes of death / survival. For a survey of the large mortality modelling literature that covers the use of Poisson models see Booth and Tickle (2008).

As we undertake a time-series analysis of the effect of COVID on mortality, we focus on a small subset of the Poisson mortality modelling literature that uses time-series data to analyse the effect of some form of discrete change on mortality. One such example of the application of a Poisson model examines the effect of rail privatisation in Great Britain from 1994 on rail accidents and fatalities (Evans, 2007a; 2007b). The approach in these two studies was to construct the counterfactual, i.e., predictions of rail accidents and fatalities that would have been expected from 1994 through to the end of the study period (2003), if Britain's railways had remained nationalised. This involved fitting a Poisson model for the number of fatal accidents, and the number of fatalities, for a time period that directly preceded the privatisation of Britain's railways. The trends from these models were then extrapolated through to 2003 to obtain predictions of the number of fatal accidents and the number of fatalities, if Britain's railways had remained nationalised. With this approach the predicted number of fatal accidents and number of fatalities can be compared to the actual numbers, and the standard errors of the differences can be calculated. There are two areas of this approach, though, that our empirical methodology for COVID enhances.

First, one would typically only forecast when observations for the dependent variable are not available, otherwise information is being discarded. We therefore include the COVID period in our sample for all our model specifications. In our baseline models, we use dummy variables to distinguish the portions of our sample that represent the COVID period and the period when the UK Government's lockdown policy was active. As we use a fixed coefficient estimator throughout, the baseline models will, of course, only yield estimates of average weekly mortality due to COVID and the lockdown. When an independent variable is continuous and when, as is the case here, the variables are not logged so that its fitted coefficient measures the effect of a marginal 
change in the variable in terms of deaths, one can still obtain per period marginal effects, which (in all likelihood) will differ in magnitude. One can do this by simply multiplying the fixed coefficient on the continuous independent variable by the ratio of the values of the independent and dependent variables for each period. When the independent variable is, for example, a dummy variable, one cannot, of course, calculate per period impacts in this way.

The advantage of the approach that Evans (2007a; 2007b) uses is that the out-of-sample differences between the predicted and actual values are for each period. This represents the second area in which we enhance previous modelling approaches: namely, that statistical inference for the differences between the predicted and actual values is for the entire out-of-sample period. To achieve this, we use a further novel empirical specification of the Poisson model to introduce additional richness that includes weekly COVID / lockdown identifier variables. These variables take a value of 1 in an individual week during the COVID / lockdown period and zero otherwise. Whereas the fitted coefficients on the dummy variables from our baseline models represent the mean weekly estimates of the impact on deaths of COVID and the lockdown, the fitted coefficients on the COVID and lockdown identifiers represent rich estimates of the weekly deviations around the relevant mean estimate.

Notwithstanding that statistical studies of COVID deaths and infections is very much an evolving area, our study differs from two types of studies that have recently emerged. The first type focuses on the transmission of COVID infections using cross sectional / panel data methods (e.g., Qiu et al., 2020, Liu et al., 2020, and Baum and Henry, 2020), where the latter draws on developments in the spatial econometrics literature (e.g., Glass et al., 2016, Baltagi et al., 2007, and Kelejian and Prucha, 2010). As a result of these studies using cross-sectional / panel data, they are very different to our study as they analyse the transmission of infections across Chinese cities (Qiu et al., 2020), approximately 100 countries / regions (Liu et al., 2020) and counties in the US (Baum and Henry, 2020). In contrast, we conduct a time series analysis at the country level. The second type of study is more similar to our approach, as this type of study corresponds to a time series analysis of infections (e.g., Wood, 2020, and Benvenuto et al., 2020). Our analysis differs from these time series studies as we focus on the issue of measuring excess deaths due to COVID.

Three key findings from our empirical analysis are as follows. First, although it has been widely reported that COVID-19 has been highly concentrated in the elderly, we find that it has been particularly concentrated in the very elderly (75-84 and 85+ years), and less so in the 65-74 age category. Second, using two sets of COVID identifiers, we find from the beginning of the two periods when we assume the lockdown was having an impact, through to the end of our study period (week ending $17^{\text {th }}$ or $24^{\text {th }}$ April 2020 - week ending $8^{\text {th }}$ May 2020), that our weekly estimates 
of COVID deaths for five cases (the total; the 75-84 and 85+ age categories; males; and females) diverge from the corresponding 5 year average excess deaths measure. Over these periods, we find that, on average per week, our estimates of COVID deaths for these five cases were (in absolute terms) considerably below the corresponding 5 year average excess deaths measure. For example, on average per week, our estimate of total COVID deaths over these periods was lower than the corresponding 5 year average excess deaths measure by $4670-4727$ deaths (54\%-63\%). For the above five cases, and in line with our hypothesis, we posit that the 5 year average excess deaths contains a large number of non-COVID deaths. Third, and relatedly, our analysis suggests that the UK's lockdown has had a net positive impact on mortalities. That is to say, it resulted in more, not less, deaths. Intuitively, this may be due to the unintended consequences of the lockdown (for example, a substantial reduction in the provision of, or access to, other forms of critical healthcare) dominating its intended consequences.

The remainder of this paper is organised as follows. Section 2 comprises three parts. In the first part we assess further the limitations of the COVID associated deaths and excess deaths methods. The second part discusses the implications of the UK Government's lockdown policy for the mortality rate. In the third part we consider the extant literature to inform the choice of variables to explain all-cause mortality in the empirical models. In section 3 we present the empirical methodology, which involves a general presentation of the specifications of the Poisson model we estimate. Section 4 provides details of the empirical specifications, data and variables, and section 5 presents and analyses the empirical results. In section 6 we conclude and discuss the scope for further work.

\section{Details of the Measures of COVID Deaths, UK Government Policy and the Relevant Literature}

\subsection{Further Assessment of the Current Measures of COVID Deaths in England and Wales}

With regard to the COVID associated deaths method, the lack of a reliable record of causality between COVID infection and the subsequent death has been the case since the method's inception. The problem, though, will be more pronounced since the previously discussed method change on April $29^{\text {th }}$ 2020. This issue is well known and is set out transparently by the ONS itself, which states: "deaths of people who have tested positively for COVID-19 could in some cases be due to a different cause." Due to this major limitation, we noted above that a consensus has formed around excess deaths being a preferred measurement approach.

Given the above consensus and, as a result, our use of an excess deaths modelling framework, we focus our attention on the excess deaths method in this discussion. Such a consensus is consistent with the wider, and long-established, academic literature regarding the measurement of the impact 
of specific events and / or disease and illness on mortality, where it is well accepted that 'direct' measures of death are inherently subject to recording error. This makes 'indirect' (in practice, typically statistical) approaches that identify excess deaths preferable. Indeed, and of particular relevance to the challenges in measuring COVID-19 deaths, this framework is commonly used to analyse drivers of variation in influenza mortality rates over time and across countries. For example, Simonsen et al. (1994) apply this approach to identify cyclical deaths relating to influenza epidemics, noting that: "influenza diagnoses are generally not laboratory confirmed... given this incomplete identification [of cause of death] an indirect approach involving statistical modelling has long been used to estimate the seasonal excess mortality attributable to influenza." Alling et al. (1981) apply a regression analysis to identify excess mortality in the US between 1968 and 1976 for this same reason. Lui and Kendell (1987) similarly use cyclical regression analysis to identify instances where mortality is 'higher than expected' (i.e., predicted by regression models) to understand the impact of influenza epidemics in the US from 1972 to 1985 . The challenges and issues associated with the measurement of mortality and an understanding of its causes are long-standing. In fact, the use of statistics in relation to these issues seems to date back to the work of William Farr (1885, pp. 166-205), who wrote reports on the 1847/48 influenza epidemic in London.

Whilst an excess deaths approach does not suffer from the above measurement limitation, we noted that reported COVID deaths under this method may nonetheless overstate true excess deaths due to the pandemic. This is because COVID may have modestly brought forward deaths that would otherwise have occurred at some future point in the year. Of relevance to this, we noted above that COVID deaths are highly concentrated in the elderly, in line with the ONS data reported in table 1. This concept is recognised in the epidemiology and public health literature and is generally referred to as mortality displacement (Kaiser et al., 2007). To illustrate this further, figures 1, 2a and $2 \mathrm{~b}$ present for England and Wales total weekly deaths, and deaths by age category, over the last 10 years. As can be seen, the COVID peak is essentially always the highest datapoint. Nonetheless, peaks are observed every year, typically in the winter (flu season). Thus, rather than the 'whole' of the COVID peak being excess deaths (which is, in essence, what any contemporaneous comparison of a weekly deaths to a 5 year average implies) it might be that only the increment between the COVID peak (April 2020) and other more typically historically observed peaks, is truly excess due to COVID.

[Insert about here Table 1: COVID associated deaths in England and Wales up to the week ending 29 May 2020]

[Insert about here Figure 1: Weekly deaths in England and Wales from 8 January 2010-15 May 2020] 
[Insert about here Figure 2a: Weekly deaths in England and Wales for three age categories from 8 January 2010-15 May 2020]

[Insert about here Figure 2b: Weekly deaths in England and Wales for a further four age categories from 8 January 2010-15 May 2020]

To explore the extent to which reported COVID deaths overstate the true number of excess deaths due to the pandemic, it is helpful to examine how unique the COVID peak in figure 1 is over a longer period of time. By reviewing monthly mortality data, as opposed to weekly above, it is possible to compare spikes in deaths over a longer period - from June 2006 to April 2020. Using this data, we have examined the extent to which the COVID peak is an outlier based on two methods. First, figure 3 shows for England and Wales the percentage difference between: (i) the peak monthly mortality in each year; and (ii) the average monthly mortality across the whole time period. Second, figure 4 presents for England and Wales the peak monthly mortality in each year as a percentage of the COVID mortality peak.

[Insert about here Figure 3: Difference for England and Wales between peak monthly mortality in a year and the long-term average monthly mortality (June 1998-April 2020)]

[Insert about here Figure 4: Peak monthly mortality in a year for England and Wales as a percentage of April 2020]

Figures 3 and 4 demonstrate that, although the COVID peak in April 2020 represents the highest mortality in recent years by some distance, there have been spikes in other years that have also far exceeded the average. Figure 3 shows that the number of deaths in April 2020 is just short of $90 \%$ higher than the average. Moreover, figure 4 reveals that there have been cases where the peak mortalities in the pre-COVID years have been well over $80 \%$ of COVID mortality.

Overall, then, although COVID mortality is particularly high, we have observed that there have been high prior peaks. This might suggest that, if deaths have in fact been 'brought forward', the current excess deaths numbers may be overstated. The plausibility of this is persuasive, when considered in parallel with the fact that the probability of dying from COVID-19 is not equal across the population, as mortalities are highly concentrated in the elderly and those with underlying medical conditions.

\subsection{UK Government Policy and its Role in Explaining COVID Mortality}

The primary objective of this paper is not to evaluate whether or not UK Government COVID policy (i.e., the lockdown) has been effective. Rather, our goal is simply to derive a more robust measure of COVID-19 excess deaths. Nonetheless, because Government policy itself may have 
impacted the mortality rate, this question cannot be ignored. Here, there are two potential impacts to consider.

First, the direct (and intended) impact of any policy intervention would typically be to spread out (and potentially reduce) overall mortalities. In the UK, the rationale for social distancing and the subsequent lockdown forwarded by both Government and its scientific advisors was framed around two concepts: (i) Mitigation (slowing, but not stopping, epidemic spread). The purpose of this is to lower peak healthcare demand in order to protect the NHS. (ii) Suppression; this would aim to reverse epidemic growth by reducing case numbers to very low levels (Ferguson et al., 2020). In practice, in the absence of a vaccine or viable treatment for COVID-19 in the near term, the second rationale and objective is unachievable. Hence, the policy choices were framed around rationale (i). Accordingly, policymakers adopted the language of 'flatten the curve' and 'protect the NHS'. In principle, rationale (i) might itself reduce total mortalities (relative to the counterfactual of no government action). For example, preventing the NHS being overwhelmed may itself achieve this. Thus, to the extent that the policy is successful, this would lead to a negative association between overall mortality and the policy response. However, unlike the previous mentioned drivers of mortality used in the literature (see subsection 2.3), the lack of historical precedent for the policy responses currently being enacted, means we have no a priori expectation as to whether said responses would achieve their stated aims.

Second, the indirect (and unintended) impact of policy interventions may well be increasing mortality. For example, under the UK lockdown, certain medical treatments were suspended by the NHS, or became harder to access. Cancer Research UK (2020) reported that: (i) cancer screening was suspended; (ii) early cancer diagnoses were materially impacted; and (iii) "despite national guidelines stating that urgent and essential cancer treatments must continue, unfortunately this is not the case in some hospitals across the UK." In addition, the British Heart Foundation (2020) reported a 50\% drop in heart attack A\&E attendances. The Stroke Association similarly has reported large reductions in hospital admissions for strokes (Clinical Services Journal, 2020).

A sense of the scale of the indirect impacts can be seen by examining data on A\&E attendance and admissions in England. Figure 5 provides a monthly time series of this from January 2011 to April 2020. As can be seen from this figure, A\&E attendance and admissions have collapsed during the COVID period, with the number for April 2020 being some 48\% lower than that for the same month in 2019.

[Insert about here Figure 5: A\&E attendance and emergency admissions in England] 
The above considerations are critical for any robust analysis of excess COVID deaths. The key points are as follows:

- The UK Government's policy response may, in principle, have two opposing impacts on mortality. Therefore, there is no null hypothesis that the policy response should reduce, or increase, mortality. The expected impact is ambiguous.

- Because it is possible that the net impact of the UK Government's policy response is negative, or neutral, it is important to control for it in any models, if possible. This is because a failure to do this may incorrectly attribute the above indirect impact (unintended consequences) of the policy to COVID-19 itself. That is to say, deaths arising from people not being treated for cancer due to lockdown would be assigned to COVID-19, rather than (correctly) ascribing these to the policy response. Here, we note that media discussion sometimes conflates this with 'collateral' impacts of COVID-19. This appears unsound, as these mortalities may not have arisen without the policy response. In any case, so long as both COVID and the policy response are parameterised in any models, one can seek to resolve this matter through evidence.

Regarding the first point above (that there is no null hypothesis that the policy response should either reduce, or increase, overall mortalities) one must further be mindful of the interpretation of the UK Government's decision to impose a lockdown on March $23^{\text {rd }}$, and the continued downward trend in deaths after that date. Specifically, one might naively conclude from such a trend that lockdown did, in fact, reduce mortalities. However, this naïve comparison of the date of a policy response and a mortality trend, in and of itself, provides no evidence that said response reduced mortalities in net terms. This is both for the reasons set out above (i.e., the number of COVID deaths being incorrectly measured), but also because it fails to take account of the time lag between infection, symptom onset, and death.

The second issue above (the time lag) is relevant to our work because, given the need to incorporate the policy response into the modelling in order to robustly measure excess COVID deaths, it is important to do so in a way that accurately reflects the epidemiology evidence. In practice, the evidence on time lags remains subject to uncertainty. However, the World Health Organization (WHO, 2020) has estimated the mean incubation period (infection to being symptomatic) to be 56 days, with a maximum of 14 days (consistent with Government quarantine advice). Lauer et al. (2020) also suggest a 5 day incubation period.

Verity et al. (2020) calculate the average time lag between symptom onset and outcomes (death or recovery). The study was based on individual case level data for patients that died from COVID19 in Hubei, China. Based on this data, they found the mean duration from symptom onset to death 
was 17.8 days (with a $95 \%$ confidence interval of 16.9 to 19.2 days). Also, in relation to the time from symptom onset to death, the WHO (2020) estimated this to range from 2 to 8 weeks.

Currently available information might therefore suggest a total time lag between infection and mortality to be around 23 days on average ( 5 days to become symptomatic; plus 18 days to death). However, the WHO figures imply a longer overall period of 40 days (5 days to become symptomatic; plus the mid-point of their 2 to 8 week range above -35 days). Combined the data therefore suggests the average total elapsed time from infection to death lies between $>3$ to $<6$ weeks.

\subsection{Literature to inform the Choice of Variables to Explain Mortality}

To develop a statistical approach to measure the incremental impact of COVID-19 on mortalities in England and Wales, it is necessary to ascertain the broader relevant factors that explain mortality. The public health, virology, epidemiology and economics literature point to a number of potentially relevant variables.

Cutler et al. (2006) set out a review of the main determinants of mortality in a historical context. Whilst they find it challenging to point definitively to specific drivers, they cite: public health; socioeconomic status; income (although they somewhat caveat this); urbanisation; and medical care and resources. Rogers (1979) finds that income distribution is consistently and strongly related to mortality. Soares (2007) explores the factors that have contributed to falling mortality rates in the developing world. The author finds weak support for income as a driver, instead finding evidence relating to public health, immunisation and knowledge transfer being more pertinent.

Consistent with the data set out in this paper, the literature discusses the cyclical nature of mortality, with mortality rates typically having a strong seasonal pattern. Nogueira et al. (2009) estimate the excess mortality associated with the influenza activity registered in Portugal between 2008 and 2009. To reflect the well-established seasonal pattern of influenza, cyclical regressions were used.

In addition, various studies have found relationships between temperature and mortality (i.e., separate to cyclical or seasonal patterns per se). This literature points to multifaceted relationships. For example, whilst 'on average' mortality may decline with temperature, extreme cold or heat wave events may increase mortality. For example, using techniques for lagged cross-correlation and spectral analyses, Cech et al. (1979) found that the mean temperature is negatively associated with mortality in Japan (i.e., less deaths as average temperatures rise). However, the authors also found that more extreme temperatures (either hot or cold) increase mortality. Consistent with this, Huynen et al. (2001) undertook a statistical analysis of the impact of ambient temperature on 
mortality in the Netherlands (1979-1997). The authors found a 'V-like' relationship, whereby mortalities were typically lower within an optimal temperature range, but increased during more extreme cold spells or heat waves. They estimated the optimal temperature in the ' $\mathrm{V}$ ' to be 16.5 degrees Celsius.

Willers et al. (2016) find that air pollution is a significant driver of mortality and that this may further be associated with peak summer temperatures (although as noted above, the literature more broadly identifies higher mortality in the winter and lower mortality in the summer).

Chaix et al. (2006) use a statistical approach to distinguish between variances in mortality due to population density and socioeconomic factors in Scania, Sweden. Using a longitudinal approach, with data from 1970 to 1993, the authors conclude that population density effects dominate. Meijer et al. (2012) examine the relationships between population density and 'area level' socio economic factors on all-cause mortality in Denmark. The authors found all-cause mortality increased with population density for all age groups. Socioeconomic factors seemed to have somewhat more of an effect on mortality amongst the elderly.

Given this study's focus on England and Wales, it is also helpful to consider recent UK specific evidence regarding drivers of mortality. Recently Murphy et al. (2019) carried out a review of the decline in the UK's rate of mortality improvements. Overall the authors consider the evidence insufficient to point to any one clear factor, or factors, as explaining the UK's stalled mortality. However, of relevance to the choice of variables to explain mortality, they consider the primary explanatory factors to be: demographic changes (aging population); international migration; declining cardiovascular disease (the causes of which are themselves multidimensional); austerity (spend on health care services); and cohort effects.

Drawing the existing evidence base together, potentially relevant variables to explain all-cause mortality can be broadly characterised as follows:

- Environmental / seasonal patterns. Data typically shows higher mortality in the winter / poor weather; and lower mortality in the summer / better weather. Temperature can impact mortality in its own right, but the relationship may be non-linear. Air pollution can be positively associated with mortality, which itself may interact with air temperature.

- Demographics. As older people are generally more likely to die, mortality rates are higher amongst populations with a higher proportion of elderly people.

- Income / socio-economic factors. Poverty / average income is sometimes cited as a contributory factor in mortality rates. The empirical evidence associated with this appears more mixed. 
- Population density. Various studies have shown a positive association between population density and all-cause mortality. This is likely to be more relevant in the context of virus related mortality, as transmission rates are intuitively increased where populations are more densely located.

- Healthcare expenditure and resources. Mortality may fall with investment in healthcare and / or where healthcare resources are increased.

- Public health. In particular, factors such as obesity, smoking, etc. may cause mortality to vary.

It is clear that the potential variables to explain all-cause mortality are complex and multidimensional. The existing literature does not, therefore, provide definitive answers as to which factors are necessarily most important for inclusion in an empirical model, which is context specific. Nonetheless, the evidence indicates that variables across the above categories should generally be given consideration.

\section{General Presentation of the Poisson Model Specifications}

For each measure of deaths that we analyse, we assume that the observed number of deaths $(y)$ follows a Poisson distribution:

$$
y \sim \text { Poisson }[\mu(m)],
$$

where the density of $y$ is determined by the conditional mean $\mu(m) \equiv E(y \mid m)$ and $m$ represents a set of determinants. We use a number of specifications of a parametric model for $\mu(m)$. The general form of our baseline specification of this model is as follows.

$$
\mu(m)=\exp \left(\beta^{\prime} \mathbf{X}+\gamma \operatorname{COVID}+\delta \text { LockD }\right),
$$

where the set of determinants $m$ are in brackets on the right-hand side.

COVID and LockD are dummy variables that take values of zero before the beginning of our COVID and lockdown periods and 1 from there onwards, respectively. The weekly time series data comprise $T$ periods, which are indexed $t \in 1, \ldots, T$. The set of time periods in our COVID period is denoted $\mathbf{J}$ and the set of time periods in our lockdown period is denoted $\mathbf{K}$. $\mathbf{K} \subset \mathbf{J}$ and the periods in $\mathbf{J}$ and $\mathbf{K}$ are indexed $j \in 1, \ldots, J$ and $k \in 1, \ldots, K . \mathbf{X}$ is a matrix that represents the observations for the set of other independent variables, and to be estimated are the parameters $\gamma$ and $\delta$ and the vector of parameters $\beta^{\prime}$. The intercept term $\left(\beta_{0}\right)$ is included within $\beta^{\prime}$ by defining the vector $\mathrm{x}_{0, t}=1$ $(\mathrm{V} t)$ in $\mathbf{X}$. The estimate of $\gamma$ is the estimate of the average weekly deaths due to COVID, and the estimate of $\delta$ is the estimate of the average weekly change in deaths due to the lockdown. Throughout our empirical analysis the parameters of the models are estimated using maximum likelihood estimation. 
The general form of our first novel specification of the model for $\mu(m)$ with identifier variables is as follows.

$$
\mu(m)=\exp \left(\beta^{\prime} \mathbf{X}+\lambda_{1} \operatorname{COVID} 1+\cdots+\lambda_{J} \operatorname{COVIDJ}+\delta \operatorname{Lock} D\right)
$$

where here we replace $\gamma$ COVID in Eq. 1 with the $J$ COVID identifier terms $\lambda_{1} \operatorname{COVID} 1+\cdots+$ $\lambda_{J}$ COVIDJ. A COVID identifier variable takes a value of 1 in the relevant week in the COVID period and zero otherwise, and $\lambda_{1}, \ldots, \lambda_{J}$ are parameters to be estimated. The mean of the estimates of $\lambda_{1}, \ldots, \lambda_{J}$ will approximate the estimate of the mean weekly deaths due to COVID $(\gamma)$ from Eq. 1. As a result, we can interpret the estimates of $\lambda_{1}, \ldots, \lambda_{J}$ as estimates of the weekly deviations of deaths due to COVID about the estimate of $\gamma$.

The general form of our second novel specification of the model for $\mu(m)$ with identifier variables is along the same lines as Eq. 2, but involves replacing $\delta$ LockD in Eq. 1 with the $K$ lockdown identifier terms $\eta_{1} \operatorname{Lock} D 1+\cdots+\eta_{K} \operatorname{Lock} D K$. A lockdown identifier variable takes a value of 1 in the relevant week in the lockdown period and zero otherwise, and $\eta_{1}, \ldots, \eta_{K}$ are parameters to be estimated. For the same reason we gave in the above discussion of Eq. 2, we can interpret the estimates of $\eta_{1}, \ldots, \eta_{K}$ as estimates of the weekly deviations about the estimate of the mean weekly change in deaths due to the lockdown $(\delta)$ from Eq. 1.

\section{Details of the Empirical Model Specifications, Data and Variables}

The following description of the data and variables is accompanied by table 2, which provides the data sources and descriptive statistics for the continuous variables. To estimate the models, we use weekly time series data for the period week ending 8 January 2010 - week ending 15 May 2020. Following on from the general presentation of the model specifications in the previous section, the measures of deaths that we analyse and assume are Poisson distributed are: total weekly all-cause deaths in England and Wales (Total Deaths); weekly all-cause deaths in England and Wales by age category (under 1 year; 1-14 years; 15-44 years; 45-64 years; 65-74 years; 75-84 years; and $85+$ years, denoted Deaths $(<1)$, Deaths (1-14), etc.); and weekly all-cause male and female deaths in England and Wales (Male Deaths and Female Deaths). ${ }^{4}$

For each of the measures of deaths, there are six empirical model specifications. These specifications differ according to the inclusion of a COVID and / or lockdown dummy variable and / or whether the specification includes COVID or lockdown identifier variables. The first two model specifications are baseline specifications, because they include COVID and lockdown

\footnotetext{
${ }^{4}$ The data for the measures of deaths are for England and Wales and not also for Scotland and Northern Ireland. This is because the Scottish and Northern Irish data are not comparable to the English and Welsh data. To illustrate, for Scotland the weekly data is total all-cause deaths and is not by age category. Scottish all-cause deaths data by age category is only available annually up until 2018. In the case of Northern Ireland, the only available data is monthly total all-cause deaths.
} 
dummy variables, and thus yield average estimates of the weekly change in deaths due to COVID and the lockdown. Details of the dummy variables in the first two model specifications are as follows.

(i \& ii). In the first baseline model specification (Base 1) we include a COVID dummy that takes a value of 1 for the week ending 6 March 2020 onwards and zero otherwise (COVID4Week); and a lockdown dummy that takes a value of 1 for the week ending 24 April 2020 onwards and zero otherwise (LockD4Week). As regards the appropriate starting point for the COVID dummy, there are two considerations. The first is that there is some uncertainty as to precisely when COVID may have impacted mortalities. Specifically, the first confirmed cases of COVID were at a hotel in York on 31 January 2020 (BBC, 2020). Allowing for the above mentioned lag between infection and mortality, this would indicate a dummy starting date of late February to mid-March (our date for the COVID dummy of the week ending 6 March represents a 4 week lag). However, modelling by the University of Oxford suggests there may have been COVID infections prior to 31 January, potentially in December or early January (Financial Times, 2020). The second consideration is a modelling one. That is to say, the impact of COVID on mortality may be difficult to identify early on in the time period. Our assumption regarding the start date for the lockdown dummy reflects the fact that the UK Government's lockdown policy became active on Monday 23 March 2020. If we then similarly allow for an appropriate (4 week) time lag to capture the gap between infection and mortality, this is consistent with the dummy taking the value of 1 from the week ending April $24^{\text {th }}$.

The coefficients on the COVID and lockdown dummies may be sensitive to our assumptions about the start of the COVID period and the lag between infection and mortality (which would determine when the lockdown policy may have first had an effect). We investigate this in the second baseline model specification (Base 2), by replacing COVIDD4Week and LockD4Week in Base 1 with a COVID dummy and a lockdown dummy that take a value of 1 from one week earlier (week ending 28 February 2020 and 17 April 2020, respectively) and zero otherwise (COVID3Week and LockD3Week). These revised assumptions that COVID3Week and LockD3Week are based on represent a closer approximation to the estimate of 23 days from infection to mortality than the 4 week assumption of COVIDD4Week and LockD4Week.

The four remaining model specifications all include novel weekly identifier variables that pertain to individual weeks in the COVID / lockdown period. The third and fourth specifications (COVID IdentV1 and COVID IdentV2) include COVID identifier variables, and the fifth and sixth specifications (LockD IdentV1 and LockD IdentV2) include lockdown identifier variables. From COVID IdentV1 and COVID IdentV2, we obtain estimates of the weekly deviations in COVID deaths around the mean weekly estimates from the Base 1 and Base 2 specifications. From LockD 
IdentV1 and LockD IdentVar2, we obtain estimates of the weekly deviations in the change in deaths due to the lockdown about the mean weekly estimates of the change from Base 1 and Base 2. Details of the identifier variables in the COVID IdentV1, COVID IdentV2, LockD IdentV1 and LockD IdentV2 model specifications are as follows.

(iii \& iv). The COVID IdentV1 model specification is the Base 1 specification with LockD4Week retained and COVID4Week replaced with 10 weekly COVID identifier variables (COVID6Mar, COVID13Mar,..,COVID8May). The COVID IdentV2 model specification is the Base 2 specification with LockD3Week retained and COVID3Week replaced with 11 weekly COVID identifier variables (COVID28Feb, COVID6Mar,...,COVID8May). A COVID identifier takes a value of 1 in the relevant week during the COVID periods for the Base 1 and Base 2 model specifications and zero otherwise. The COVID periods for Base 1 and Base 2 actually span 11 weeks and 10 weeks, but to fit the model we must drop one COVID identifier in the lockdown period, which is for the last week. This is because, although the COVID identifiers account for individual weekly effects, by dropping one during the lockdown period the COVID identifiers during this period will not collectively account for the same effect as LockD4Week or LockD3Week.

(v \& vi). The LockD IdentV1 model specification is the Base 1 specification with COVID4Week retained and LockD4Week replaced with 4 weekly lockdown identifier variables (LockD24Apr,...,LockD15May). The LockD IdentV2 model specification is the Base 2 specification with COVID3Week retained and LockD3Week replaced with 5 weekly lockdown identifier variables (LockD17Apr,..,LockD15May). A lockdown identifier takes a value of 1 in the relevant week during the lockdown periods for Base 1 and Base 2 and zero otherwise. We do not need to drop one of the lockdown identifiers to estimate LockD IdentV1 and LockD IdentV2 because the assumed COVID period (as represented by COVID 4 Week or COVID 3 Week) is longer than the assumed lockdown period.

Before we discuss the continuous independent variables, we note that we include two further dummy variables to take account of the variation in mortality according to the time of year. The two dummy variables (Summer and Winter) we include are for the summer (Jun, Jul and Aug) and winter (Dec, Jan and Feb) months.

Since data for the measures of deaths are rather high frequency, we are faced with some decisions regarding the independent variables when the available data are of a lower frequency and / or are for a different time period. In the case of the percentages of the UK population by age (1 year, 2 years, ..., 90+ years), the ONS data is annual and the most recent data are for 2019. We include as an independent variable in all the models the percentage of the population that is $90+$ years 
(Share90+) because COVID deaths have been highly concentrated in the elderly. Also, any changes over time in population percentages that relate to the elderly that are aged below 90 will conceivably be positively correlated with Share90+. This is because the quality of healthcare of the elderly and their quality of life are likely to be positively correlated across elderly age categories. We also assume that the annual Share90+ percentage applies to each week in the relevant year. ${ }^{5}$ This is with the exception of the weeks in 2020 , for which a Share $90+$ observation is not available. In this case we assume the 2019 Share $90+$ observation applies. In normal times this would not be unreasonable, because Share90+ is not likely to differ greatly between consecutive years. Of course, 2020 does not represent normal times because the actual annual Share $90+$ for 2020 will be affected by COVID-19 deaths. That said, the effect of COVID deaths on the actual Share $90+$ for 2020 is a source of endogeneity that we circumvent by assuming that our 2020 observation is equal to that for 2019.

If the data for Share $90+$ was of a higher frequency, such as weekly, this variable would be endogenous in the Deaths(85+) model, and also possibly the Deaths(75-84) model, because Deaths(85+) and Deaths(75-84) are likely to be positively correlated. As Share90+ is constant in our dataset across the weeks in any year, it is not therefore endogenous in the Deaths(85+) and Deaths(75-84) models (because no weekly observation for either of these measures of deaths could feasibly influence the annual measure of Share $90+$ ). Moreover, using the same approach as we use to construct the Share $90+$ variable, we construct average weekly population density across local authorities in England and Wales (PopDen measured as people per sq. $\mathrm{km}$ ). We use this approach because, first, the data is available annually up to and including 2019 and, second, population density will be largely stable over the course of a year.

The available temperature data for England and Wales are mean monthly measures for each country. We therefore constructed a mean monthly temperature measure across the two countries (weighted by their annual populations). ${ }^{6} \mathrm{We}$ then assumed that this weighted mean applied to each week in the relevant month (Temp). This is because only the frequency of the available data differed from our sample and not the time period. We therefore consider that the difference between using our weighted mean monthly temperature at the weekly level, and the actual mean temperature for the week, is unlikely to be material. It turns out that this does not appear to impact

\footnotetext{
${ }^{5}$ Although this assumption may not be particularly realistic because relatively more (fewer) elderly people die in the winter (summer) leading to a decline (rise) in Share90+, it turns out that it does not materially impact our estimated models. This is likely to be because Share $90+$ represents a very small percentage of the population. Any changes in Share $90+$ therefore are likely to be relatively small and will not constitute big departures from our assumption that the annual Share $90+$ percentage applies to each week in the relevant year. Moreover, a big advantage of making this assumption is that Share90+ is exogenous, which would not be the case if we used higher frequency data where in each year Share $90+$ decreased (increased) in the winter (summer).

${ }^{6}$ Population data for England and Wales was not yet available for 2020. We therefore estimated the 2020 data by extrapolating.
} 
on the estimated models, which is likely because it is marked changes in mean temperature that lead to material changes in deaths, and not incremental temperature changes.

We explored the possibility of including independent variables to account for air pollution, government healthcare expenditure and public health (i.e., the prevalence of obesity and smoking). Ultimately, we concluded that it was not feasible to do so. This was because we could not adopt the same approach to the inclusion of these variables as we do for Share90+ and PopDen. Like Share90+ and PopDen, the data for air pollution, government healthcare expenditure and public health are available annually and are collected with a time lag, and, as a result, the data for 2019 and 2020 are not yet available. Extrapolating to obtain estimates of the 2019 and 2020 observations for these variables is not the issue. Doing so would still leave the problem of the apportionment of the annual data for these variables to the weeks in our sample. This is because with these variables we cannot reasonably assume, as we did when constructing the Share90+ and PopDen variables, that the weekly observations remain reasonably fixed over the course of a year. Air pollution, for example, will vary according to weekly economic activity.

The mean temperature variable and air pollution, however, are related. As we noted in subsection 2.3, although temperature can impact mortality in its own right, the relationship may be non-linear. This is, in part, because air pollution can be positively associated with mortality, which is an effect that may interact with air temperature. We attempt to account (to some extent) for this nonlinearity, and the effect of air pollution on mortality, by including Temp ${ }^{2}$.

It is natural to think about using real GDP per capita as the income measure in a country level analysis of mortality. As this variable is available annually, we are faced with the same issue as we discussed above for the data on air pollution, government healthcare expenditure and public health. Namely, if we were to use real GDP per capita, there would be the problem of the apportionment of the annual data to the weeks in our sample. For real GDP per capita, one cannot reasonably assume that the weekly observations are reasonably fixed over the course of a year, so that the annual data can be apportioned equally to each week. To circumvent the difficulty involved in appropriately apportioning this annual data to weeks and to use a measure of economic activity that has the desirable feature of weekly variation, we use the 4 week rolling average of the FTSE All-Share Index (FTSE All). We use the FTSE All-Share Index (rather than the FTSE 100 Index / FTSE 250 Index) as it provides a greater coverage of market capitalisation (98-99\%). This is evident as it is the aggregation of the FTSE 100, 250 and Small Cap indexes.

[Insert about here Table 2: Data sources and summary statistics] 


\section{Empirical Results and Discussion}

\subsection{Estimated Models}

In table 3 we present the fitted Base 1 models for Total Deaths, Male Deaths and Female Deaths, all of which contain the COVID4Week and LockD4Week dummy variables. In table 4 we present further fitted Base 1 models for deaths by age category (under 1 year; 1-14 years; 15-44 years; 45 64 years; $65-74$ years; $75-84$ years; and $85+$ years). The models that correspond to those in tables 3 and 4, but which are Base 2 models and thus include the COVID3Week and LockD3Week dummy variables, are presented in the Appendix in tables A1 and A2, respectively. ${ }^{7}$ In line with the focus of our attention, we commence the discussion of the fitted models by analysing the estimates of the COVID and lockdown parameters. In this subsection we provide an overall discussion of the results for these parameters that relates to their signs, significance and (broadly speaking) their relative sizes. We adopt this approach because in the next subsection we discuss the magnitudes of these parameters in detail. The coverage of the estimated models in this subsection closes with a discussion of the fitted coefficients on the other independent variables that are common to all the reported models.

[Insert about here Table 3: Total, male and female Base 1 models with COVID4Week and LockD4Week dummy variables]

[Insert about here Table 4: Age category Base 1 models with COVID4Week and LockD4Week dummy variables]

We can see from table 3 that the coefficient on COVID4Week in the Total Deaths model is positive, as we would expect, and significant at the $0.1 \%$ level. We do not take logs of the variables in our models, so that the coefficients are in terms of deaths, which enriches the interpretation. In table 3, therefore, the estimated COVID4Week parameter in the Total Deaths model suggests that, on average, there were 3,497 deaths per week during the assumed COVID period for Base 1 models (week ending 6 March 2020 to the week ending 15 May 2020). First impressions suggest this estimate is reasonable, although we postpone a detailed discussion of the actual fitted magnitudes of the COVID and lockdown parameters until the next subsection. The estimated COVID parameters in the Male Deaths and Female Deaths models in table 3 are also positive, significant at the $0.1 \%$ level, and are of a substantial magnitude. These parameters though are, as we would expect, smaller than the aforementioned COVID parameter in the Total Deaths model in the same table.

As we discussed above, the expected sign of a lockdown parameter is ambiguous. The coefficients on LockD4Week in the Total Deaths, Male Deaths and Female Deaths models in table 3 are

\footnotetext{
${ }^{7}$ To aid comparisons we again report the corresponding Total Deaths model in tables 4 and A2.
} 
positive, significant at the $0.1 \%$ level and non-negligible. We therefore suggest that the increase in deaths due to the unintended consequences of the lockdown policy is clearly dominating the intended death prevention effect of the policy. It is evident from table 3 that the estimated COVID4Week parameter in the Male Deaths model is greater than that in Female Deaths model, which may reflect a higher COVID mortality risk for males.

Table 3 also reveals that the estimated COVID4Week parameter in the Total Deaths and Male Deaths models is greater than the corresponding LockD4Week parameter, while the opposite is the case in the Female Deaths model. Table 4 indicates that the latter is due to the relatively large increase in deaths in the $85+$ age category during the lockdown. This is evident as the Deaths(85+) model is the only one in table 4 where the COVID4Week and LockD4Week parameters are both significant (and the latter is larger than the former). More specifically, when we estimate the same Base 1 model specification for Male Deaths(85+) and Female Deaths(85+), it is clear from these (unreported) models that it is the female $85+$ model where the LockD4Week parameter is (substantially) greater than that for the COVID4Week variable. ${ }^{8}$ The relative size of these two parameters suggests that the unintended consequences of the lockdown, such as a lack of appropriate healthcare during this period, were particularly felt by females in the $85+$ age category.

We can see from table A1 that when we employ the Base 2 model specification, the COVID 3 Week (LockD3Week) parameter is positive, non-negligible and significant at the $0.1 \%$ level in the models for Total Deaths, Male Deaths and Female Deaths. That said, the magnitude of the COVID3Week (LockD3Week) parameter in each of these models is notably smaller (larger) than the COVID4Week (LockD4Week) parameter in the corresponding model in table 3. To illustrate, the COVID3Week parameter in the Total Deaths model indicates that, on average, weekly total deaths due to COVID were $23 \%$ lower than the COVID4Week parameter suggests.

In addition, whereas we noted above that the Female Deaths model is the only case in table 3 where the LockD4Week parameter is larger than the COVID4Week parameter, we observe that the LockD3Week parameter is greater than the COVID3Week parameter for all three models in table A1. Such a contrast between the difference in the magnitudes of the LockD3Week and COVID3Week parameters, vis-à-vis the difference in the magnitudes of the LockD4Week and COVID4Week parameters, does indeed demonstrate that the COVID and lockdown parameter estimates are sensitive to the assumed start dates of the two periods in the modelling. As a result of the COVID3Week and LockD3Week variables bringing the start dates of the two periods forward, the aforementioned contrast arises. This is because we allow less time for the infection to have an impact, which reduces the estimate of COVID deaths, while at the same time we extend

\footnotetext{
${ }^{8}$ The unreported Male Deaths(85+) and Female Deaths(85+) models are available from the corresponding author upon request.
} 
the lockdown period (which is consistent with more deaths being attributed to its unintended consequences).

The estimates of the COVID and lockdown parameters in table 4 (A2) indicate which age categories are the main drivers of the COVID and lockdown results from the Total Deaths model in table 3 (A1). From tables 4 and A2 we can see that COVID deaths and lockdown deaths were, as we expected, highly concentrated in the 75-84 and 85+ age categories. Since COVID mortality has been highly concentrated in the elderly and, all else equal, there is a higher risk of ill-health in the elderly, we might have expected COVID deaths and lockdown deaths in the 65-74 age group to make up a larger proportion of total COVID deaths and total lockdown deaths. To illustrate, tables 4 and A2 indicate that COVID (lockdown) deaths in the 65-74 group are of the order of 36\%-43\% (13\%-39\%) of COVID (lockdown) deaths in the 75-84 and 85+ categories. We therefore conclude that COVID deaths and deaths in the lockdown due to its unintended consequences have been highly concentrated in the very elderly.

In table 5 we present the fitted COVID IdentV1 models for Total Deaths, Male Deaths and Female Deaths, all of which contain the LockD4Week dummy variable and 10 weekly COVID identifier variables. Table 6 presents further COVID IdentVI models for deaths by age categories. The models that correspond to those in tables 5 and 6, but which are COVID IdentV2 models and hence include the LockD3Week dummy and 11 weekly COVID identifier variables are presented in the Appendix in tables A3 and A4, respectively. In table 7 we presented the fitted LockD IdentV1 models for Total Deaths, Male Deaths and Female Deaths, all of which contain the COVID4Week dummy variable and 4 weekly lockdown identifier variables. Table 8 presents additional LockD IdentV1 models for deaths by age categories. The models that correspond to those in tables 7 and 8, but which are LockD IdentV2 models and therefore contain the COVID3Week dummy variable and 5 weekly lockdown identifier variables are presented in the Appendix in tables A5 and A6, respectively.

[Insert about here Table 5: Total, male and female COVID IdentV1 models with the LockD4Week dummy variable and 10 weekly COVID identifier variables]

[Insert about here Table 6: Age category COVID IdentV1 models with the LockD4Week dummy variable and 10 weekly COVID identifier variables]

[Insert about here Table 7: Total, male and female LockD IdentV1 models with the COVID4Week dummy variable and 4 weekly lockdown identifier variables]

[Insert about here Table 8: Age category LockD IdentV1 models with the COVID4Week dummy variable and 4 weekly lockdown identifier variables] 
We postpone a detailed analysis of the magnitudes of the fitted coefficients on the COVID and lockdown identifier variables until the next subsection. Here, the discussion of these coefficients is at the more general level of their signs and significance. In this regard we highlight two salient features of the results for the COVID and lockdown identifier parameters. First, we report widespread evidence of significant COVID and lockdown identifier parameters that are often nonnegligible, which clearly justifies our decision to estimate models that include identifier variables.

Second, although, as we would expect, many of the significant identifier parameters have the same sign as the dummy variable parameter they collectively replace in the Base 1 or Base 2 model specification, there are a small number of identifier parameters that are odds with the corresponding dummy variable parameter (which is a mean estimate). Thus, these provide some interesting information about departures from the mean. ${ }^{9}$ The key examples are as follows: the non-negligible negative COVID8May identifier parameters in tables 5 and 6 (A3 and A4) are at odds with the non-negligible positive COVID4Week (COVID3Week) dummy variable parameters in tables 3 and 4 (A1 and A2); and the non-negligible negative LockD8May identifier parameters in tables 7 and 8 are at odds with the non-negligible positive LockD4Week dummy variable parameters in tables 3 and 4. We suggest that we observe negative coefficients on the COVID8May variable as this is the latest COVID identifier in our sample, and at some point there will be signs of COVID mortality beginning to dissipate (or, in other words, mortality exhibiting signs of a return to its normal pattern). This effect also seems to be reinforced by the negative coefficients on the corresponding lockdown identifier, which suggest that the lockdown saved lives (in net terms) in the week ending 8 May 2020.

As a final point in this general discussion of the estimated models, we make a case for using the models in the next subsection to obtain a better estimate of excess deaths due to COVID. This case involves providing a justification for our models on the grounds they are well-specified. From tables 4 and A2, we can see that there are a lot of significant coefficients in the models for deaths in the elderly age groups, which is what we would expect because there is a higher risk of mortality in the elderly. In addition, for the variables that are common across all the reported models and where it is clear that the variable should have a positive / negative impact, we consistently report coefficients with the expected signs. This is evident, as we consistently report positive coefficients on the PopDen and Winter variables, and negative coefficients on the FTSE All and Temp variables.

\footnotetext{
${ }^{9}$ The COVID identifiers in tables 5 and 6 (A3 and A4) replace the COVID4Week (COVID3Week) dummy in tables 3 and 4 (A1 and A2), respectively; and the lockdown identifiers in tables 7 and 8 (A5 and A6) replace the LockD4Week (LockD3Week) dummy in tables 3 and 4 (A1 and A2), respectively.
} 
The coefficients on the COVID4Week and LockD4Week (COVID3Week and LockD3Week) dummy variables in tables 3 and 4 (A1 and A2) are the estimates of the average weekly excess deaths due to COVID and the average weekly change in deaths due to the lockdown. The significant such estimates are represented by the red lines in figures 6-9.

The coefficients on the COVID4Week (COVID3Week) dummy variable in tables 7 and 8 (A5 and A6) are also estimates of the average weekly excess deaths due to COVID. These estimates are good approximations of the corresponding estimates in tables 3 and 4 (A1 and A2), respectively, which indicates that the like-for-like results from the different model specifications are robust. To illustrate, the significant coefficients on the COVID4Week variable in the Total Deaths, Male Deaths and Female Deaths models in table 3 are 3,497, 2,073 and 1,428, whereas the corresponding significant estimates in table 7 are 3,518, 2,084 and 1,437. For the purposes of comparability, in figures 6 and 7 we use the estimates of the average weekly excess deaths due to COVID from tables 3, 4, A1 and A2, which is for the following reason.

In contrast, the coefficients on the LockD4Week (LockD3Week) dummy variable in tables 5 and 6 (A3 and A4) are not estimates of the average weekly change in deaths due to the lockdown. This is because the fitted models in these tables do not include the COVID identifier for the last week in our sample, i.e., COVID15May. As a result, the coefficients on the LockD4Week (LockD3Week) dummy variable in tables 5 and 6 (A3 and A4) are picking up the effect of the omitted COVID15May identifier. This explains why the fitted coefficients on the LockD4Week (LockD3Week) variable in tables 3 and 4 (A1 and A2), which are estimates of the average weekly change in deaths due to the lockdown, differ from the fitted coefficients on LockD4Week (LockD3Week) in the corresponding models in tables 5 and 6 (A3 and A4). For example, in table 3 the significant coefficient on the LockD4Week variable in the Total Deaths model is 2,693, compared to the corresponding significant coefficient of 4,624 in table 5. ${ }^{10}$ As we therefore use the estimates of average weekly deaths due to the lockdown from tables 3, 4, A1 and A2 in figures 8 and 9, for the purposes of comparability, we also use from the same tables the estimates of average weekly excess deaths due to COVID in figures 6 and 7.

\footnotetext{
${ }^{10}$ We noted in the previous subsection that the negative fitted coefficients on the COVID8May identifier in tables 5 and A3 may be interpreted as a sign of COVID mortality beginning to dissipate. The positive significant coefficients on the LockD4Week (LockD3Week) variable in table 5 (A3), however, are substantially larger than the corresponding significant positive coefficients in table 3 (A1). This suggests that this dissipation was short-lived or there was some volatility at the start of the dissipation. This is because it would appear that the large positive significant coefficients on the LockD4Week (LockD3Week) variable in table 5 (A3) are picking up the large positive COVID deaths in the week ending 15 May 2020. We explored this by replacing the LockD4Week dummy in the Total Deaths model in table 5 with a COVID15May identifier and by replacing the LockD3Week dummy in the Total Deaths model in table A3 with the same identifier. In line with are above suggestion we find that in both cases the COVID15May parameter is positive, large and significant. These two models with a full complement of COVID identifiers are not reported but are available from the corresponding author upon request.
} 
The lockdown and COVID identifier parameters in tables 5-8 (A3-A6) can be interpreted as weekly deviations around the corresponding mean estimate in tables 3 and 4 (A1 and A2). This is evident because from a model with the full complement of identifiers, such as the Total Deaths model in table 7 , the average of the lockdown identifier parameters $(2,725)$ is a good approximation of the coefficient on the lockdown dummy in the Total Deaths model in table 3 $(2,693)$. In figure 6 (7) for the models with significant COVID parameters in tables 3 and 4 (A1 and A2), we present the average weekly excess COVID deaths that these parameters represent; the weekly deviations from the average estimates that the COVID identifier parameters represent; and the widely reported 5 year average measure of excess deaths. In figure 8 (9) for the models with significant lockdown parameters in tables 3 and 4 (A1 and A2), we present the average weekly change in deaths due to the lockdown that these parameters represent; and the weekly deviations from the average estimates that the lockdown identifier parameters represent.

[Insert about here Figure 6: Excess deaths due to COVID from the Base 1 and COVID IdentV1

$$
\text { models] }
$$

[Insert about here Figure 7: Excess deaths due to COVID from the Base 2 and COVID IdentV2 models]

[Insert about here Figure 8: Change in deaths due to the lockdown from the Base 1 and LockD IdentV1 models]

[Insert about here Figure 9: Change in deaths due to the lockdown from the Base 2 and LockD IdentV2 models]

The four key findings from figures 6-9 are important and clear. (i) It is evident from figures 6 and 7 that our predictions of excess deaths due to COVID in the 15-44 age category, approximate very well over our entire sample to the corresponding 5 year average excess deaths. (ii) From the same figures, we can see that our predictions of excess deaths due to COVID as a total and for males, females and the other four age categories in the figures, approximate the corresponding 5 year average excess deaths very well until the beginning of our lockdown periods. (iii) We can see that when we continue our predictions in (ii) over our lockdown periods, these predictions are below the levels of the corresponding 5 year average excess deaths. This is particularly so for our predictions of excess deaths: in totality; for males; females; and the 75-84 and 85+ age categories. For these cases, and in line with our hypothesis in the opening section, we suggest that the 5 year average excess deaths contains a large number of non-COVID deaths. (iv) From figures 8 and 9 we can see that over the course of our lockdown periods, the increase in mortality due to the dominant unintended consequences of the lockdown policy tends to decline, which leads to a small amount of evidence where the policy has the desired effect and reduces mortality. Over the lockdown period as a whole, however, the policy has increased mortality. 
To elaborate on (iii) above, in tables 9 and 10 we present from the beginning of our lockdown periods, the average weekly difference between the 5 year average excess deaths and our estimates of COVID deaths from figures 6 and 7. Among other things, these tables reveal the specific values from these figures of the large average gaps between the blue lines and the dashed black lines for: the total; males; females; and the 75-84 and 85+ age categories. For example, tables 9 and 10 report that, on average per week, our estimates of COVID deaths in totality and for the $85+$ category are - 4670-4727 deaths (54\%-63\%) and 2335-2361 deaths (57\%-64\%) lower than the 5 year average excess deaths.

[Insert about here Table 9: 5 year mean weekly excess deaths versus mean weekly excess deaths due to COVID from the COVID IdentV1 models - week ending 24 April 2020-week ending 8

May 2020]

[Insert about here Table 10: 5 year mean weekly excess deaths versus the mean weekly excess deaths due to COVID from the COVID IdentV2 models - week ending 17 April 2020-week ending 8 May 2020]

\section{Concluding Remarks and Further Work}

This paper makes progress on developing empirical methods that can more robustly quantify COVID deaths. Specifically, by controlling for other factors that affect mortality, and by employing a novel approach to the statistical modelling by including identifier variables, we are better able to identify mortalities due to COVID.

The finding that the Government's lockdown policy increased mortality in net terms is likely to be of particular interest, but is unremarkable. As noted, the lockdown rationale was not to reduce mortality in the first place, but rather, to 'flatten the curve'. When one then considers the possibility that the policy might cause mortalities through unintended consequences, such a result is entirely plausible. This suggests further avenues of work to better understand the nature of the unintended consequences may be important. For example, unintended consequences may relate to a reduction in the provision of, and access to, other forms of critical healthcare. They might also include individuals choosing not to access healthcare during the lockdown, say because they perceived the risk from COVID to be greater than other critical medical conditions. What is directly observable, however, is a large reduction in said critical healthcare during the lockdown period. This has profound implications for both future policymaking and behavioural science. On the former, it raises questions about the merits of blanket policy responses that contrast with material variances in the actual risk by age and demographics. On the latter, one must consider whether the design of Government communications to encourage citizens to comply with lockdown in the first place inadvertently drive other, more harmful, behaviours. 


\section{Declarations}

Funding: Not Applicable; Conflicts of interests / Competing interests: None; Availability of data and material: Yes - from the corresponding author upon request / will be made publicly available via the journal; Code availability: Stata estimation commands are available from the corresponding author upon request.

\section{References}

Alling, D. W., W. C. Blackwelder and C. H. Stuart-Harris (1981): 'A study of excess mortality during influenza epidemics in the United States, 1968-1976'. American Journal of Epidemiology, vol. 113, pp. 30-43.

Baltagi, B.H., S. H. Song, B. C. Jung. and W. Koh (2007): 'Testing for serial correlation, spatial autocorrelation and random effects using panel data'. Journal of Econometrics, vol. 140, pp. 5-51.

Baum, C. F. and M. Henry (2020): 'Socioeconomic factors influencing the spatial spread of COVID-19 in the United States'. Boston College Working Paper 1009.

Benvenuto, D., M. Giovanetti, L. Vassallo, S. Angeletti and M. Ciccozzi (2020): 'Application of the ARIMA model on the COVID-2019 epidemic dataset'. Data in Brief, vol. 29, Article 105340.

Booth, H. and L. Tickle (2008): 'Mortality modelling and forecasting: A review of methods'. Annals of Actuarial Science, vol. 3, pp. 3-43.

BBC (2020): 'Coronavirus: Two cases confirmed in the UK'. 31 $31^{\text {st }}$ January 2020. Available at: https://www.bbc.co.uk/news/health-51325192

British Heart Foundation (2020): 'Lives at risk due to 50\% drop in heart attack A\&E attendances'. $9^{\text {th }}$ April 2020. Available at:

https://www.bhf.org.uk/what-we-do/news-from-the-bhf/news-archive/2020/april/drop-in-heart-attack-patientsamidst-coronavirus-outbreak

Cancer Research UK (2020): 'How coronavirus is impacting cancer services in the UK'. 21 $1^{\text {st }}$ April 2020. Available at:

https://scienceblog.cancerresearchuk.org/2020/04/21/how-coronavirus-is-impacting-cancer-services-in-the-uk/

Cech, I., M. H. Smolensky, R. Lane, H. Nagata, Y. Takahashi and T. Morimoto (1979): 'Day-to-day and seasonal fluctuations of urban mortality in Kyoto, Japan'. International Journal of Biometeorology, vol. 23, pp. 89-105. Chaix, B., M. Rosvall, J. Lynch and J. Merlo (2006): 'Disentangling contextual effects on cause-specific mortality in a longitudinal 23-year follow-up study: impact of population density or socioeconomic environment?' International Journal of Epidemiology, vol. 35, pp. 633-43.

Coelho, E. and L. C. Nunes (2011): 'Forecasting mortality in the event of a structural change'. Journal of the Royal Statistical Society: Series A (Statistics in Society), vol. 174, pp. 713-736.

Clinical Services Journal (2020): 'Stroke concern rising amid COVID-19 crisis warns top charity'. $6^{\text {th }}$ April 2020. Available at:

https://www.clinicalservicesjournal.com/story/32340/stroke-concern-rising-amid-covid-19-crisis-warns-top-

charity

Cutler, D., A. Deaton and A. Lleras-Muney (2006): 'The determinants of mortality'. Journal of Economic Perspectives, vol. 20, pp. 97-120.

EuroMOMO (2020): 'Rationale'. Available at:

https://www.euromomo.eu/how-it-works/rationale

Evans, A. W. (2007a): 'Rail safety and rail privatisation in Britain'. Accident Analysis \& Prevention, vol. 39, pp. 510-23.

Evans, A. W. (2007b): 'Rail safety and rail privatisation'. Journal of the Royal Statistical Society-Significance, vol. 4, pp. 15-18.

Farr, W (1885): Vital statistics: a memorial volume of selection from the reports and writings of William Farr'. London: The Sanitary Institute of Great Britain

Ferguson, N. M., D. Laydon, G. Nedjati-Gilani, N. Imai, K. Ainslie, M. Baguelin et al. (2020): 'Report 9: Impact of non-pharmaceutical interventions (NPIs) to reduce COVID-19 mortality and healthcare demand.' Imperial College COVID-19 Response Team.

Financial Times (2020): 'Coronavirus may have infected half of UK population - Oxford study'. 24 March 2020. Glass, A. J., K. Kenjegalieva and R. C. Sickles (2016): 'A spatial autoregressive stochastic frontier model for panel data with asymmetric efficiency spillovers'. Journal of Econometrics, vol. 190, pp. 289-300.

Health Foundation (2020): 'Understanding excess mortality'. $6^{\text {th }}$ May 2020.

HM Government (2020): 'Coronavirus (COVID-19) cases in the UK - About the data'. Available at: 
https://coronavirus.data.gov.uk/about\#covid-19-associated-deaths

Huynen, M. M. T. E., P. Martens, D. Schram, M. P. Weijenberg and A. E. Kunst (2001): 'The impact of heat waves and cold spells on mortality rates in the Dutch population'. Environmental Health Perspectives, vol. 109, pp. 463-70.

Kaiser, R., A. Le Tertre, J. Schwartz, C. A. Gotway, W. H. Daley and C. H. Rubin (2007): 'The effect of the 1995 heat wave in Chicago on all-cause and cause-specific mortality'. American Journal of Public Health, vol. 97, Supplement 1, pp. S158-S162.

Kelejian, H. H. and I. R. Prucha (2010): 'Specification and estimation of spatial autoregressive models with autoregressive and heteroskedastic disturbances'. Journal of Econometrics, vol. 157, pp. 53-67.

Lauer, S. A., K. H. Grantz, Q. Bi, F. K. Jones, Q. Zheng, H. R. Meredith, A. S. Azman, N. G. Reich and J. Lessler (2020): 'The incubation period of coronavirus disease 2019 (COVID-19) from publicly reported confirmed cases: estimation and application.' Annals of Internal Medicine, vol. 172, pp. 577-582.

Liu, L., H. R. Moon and F. Schorfheide, (2020): 'Panel forecasts of country-level Covid-19 infections.' NBER Working Paper No. 27248.

Lui, K-J. and A. P. Kendal (1987): 'Impact of influenza epidemics on mortality in the United States from October 1972 to May 1985'. American Journal of Public Health, vol. 77, pp. 712-6.

Meijer, M., A. M. Kejs, C. Stock, K. Bloomfield, B. Ejstrud and P. Schlattmann (2012): 'Population density, socioeconomic environment and all-cause mortality: A multilevel survival analysis of 2.7 million individuals in Denmark'. Health \& Place, vol. 18, pp. 391-9.

Michener, R. and C. Tighe (1992): 'A Poisson regression model of highway fatalities'. American Economic Review, vol. 82, pp.452-456.

Murphy, M., M. Luy and O. Torrisi (2019). 'Stalling of mortality in the United Kingdom and Europe: an analytical review of the evidence'. LSE Department of Social Policy Working Paper 11-19.

Nogueira, P. J., B. Nunes, A. Machado, E. Rodrigues, V. Gómez, L. Sousa and J. M. Falcão (2009): 'Early estimates of the excess mortality associated with the 2008-9 influenza season in Portugal'. Euro Surveillance, vol. 14 (18).

ONS (2020): 'Provisional weekly death registrations and percentage of excess death for selected cases, England and Wales: 2020'. Available at:

https://www.ons.gov.uk/peoplepopulationandcommunity/birthsdeathsandmarriages/deaths/adhocs/11683 provis ionalweeklydeathregistrationsandpercentageofexcessdeathforselectedcausesenglandandwales2020

Regidor, E., F. Vallejo, J. A. Tapia Granados,, F. J. Viciana-Fernández, L. de la Fuente and G. Barrio (2016): 'Mortality decrease according to socioeconomic groups during the economic crisis in Spain: a cohort study of 36 million people'. The Lancet, vol. 388, pp. 2642-2652.

Qiu, Y., X. Chen and W. Shi (2020): 'Impacts of social and economic factors on the transmission of coronavirus disease 2019 (COVID-19) in China'. Forthcoming in the Journal of Population Economics.

Rodgers, G. B. (1979): 'Income and inequality as determinants of mortality: an international cross-section analysis'. Population Studies- A Journal of Demography, vol. 33, pp. 343-51.

Simonsen, L., M. J. Clarke, G. D. Williamson, D. F. Stroup, N. H. Arden and L. B. Schonberger (1997): 'The impact of influenza epidemics on mortality: introducing a severity index'. American Journal of Public Health, vol. 87, pp. 1944-50.

Sekhri, S. and A. Storeygard (2014): 'Dowry deaths: Response to weather variability in India'. Journal of Development Economics, vol. 111, pp.212-223.

Soares, R. R. (2007): 'On the determinants of mortality reductions in the developing world'. Population and Development Review, vol. 33, pp. 247-87.

Verity, R., L. C. Okell, I. Dorigatti, P. Winskill, C. Whittaker, N. Imai, et al. (2020): 'Estimates of the severity of coronavirus disease 2019: a model-based analysis'. Forthcoming in Lancet Infectious Diseases.

Willers, S. M., M. F. Jonker, L. Klok, M. P. Keuken, J. Odink, S. van den Elshout, C. E. Sabel, J. P. Mackenbach and A. Burdorf (2016): 'High resolution exposure modelling of heat and air pollution and the impact on mortality'. Environment International, vols. 89-90, pp. 102-9.

Wood, S. N. (2020): 'Did COVID-19 infections decline before UK lockdown?' Mimeo.

World Health Organization (2020): Report of the WHO-China Joint Mission on Coronavirus Disease 2019 (COVID-19). 
Figure 1: Weekly deaths in England and Wales from 8 January 2010-15 May 2020

25000

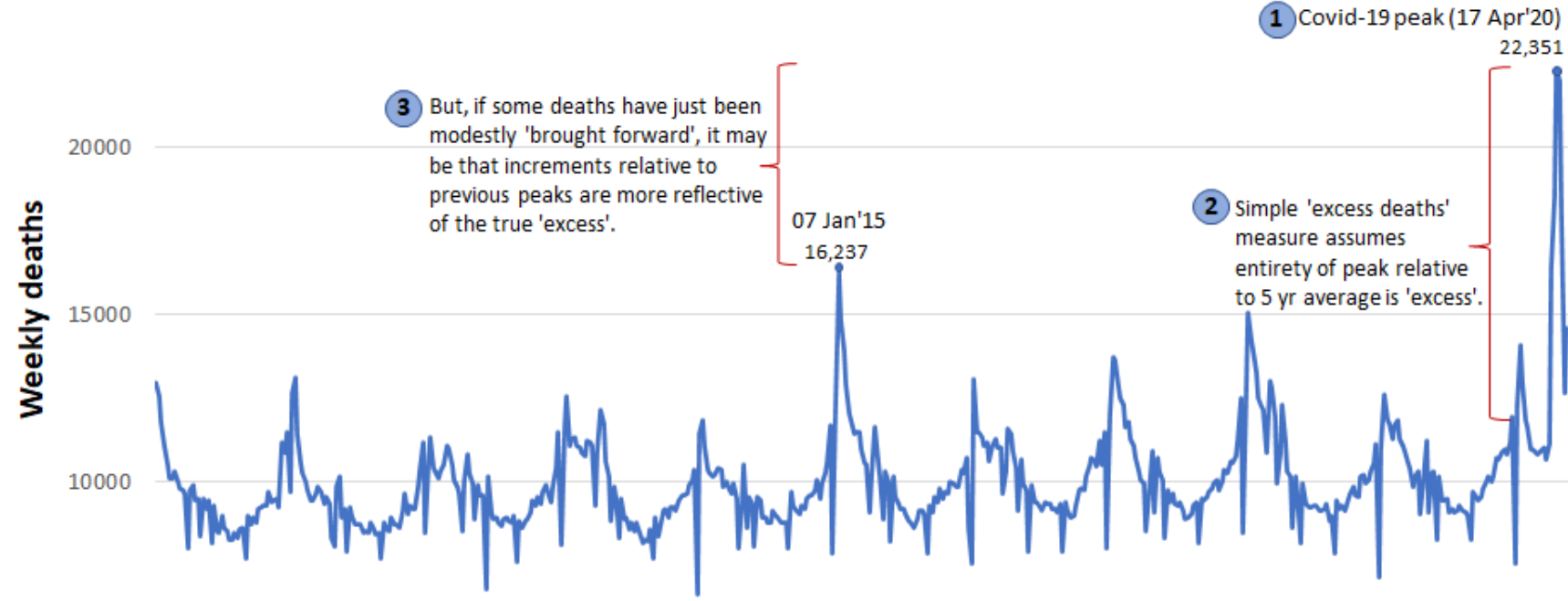

5000

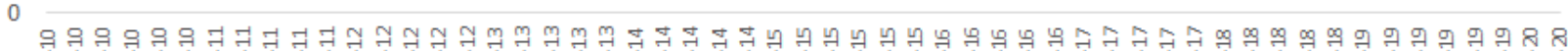

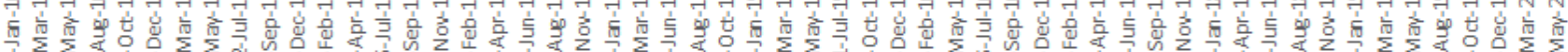

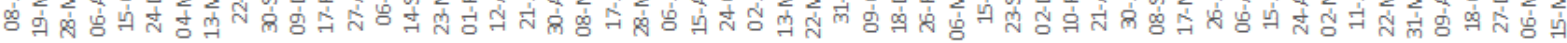

Figure 2a: Weekly deaths in England and Wales for three age categories from 8 January 2010-15 May 2020

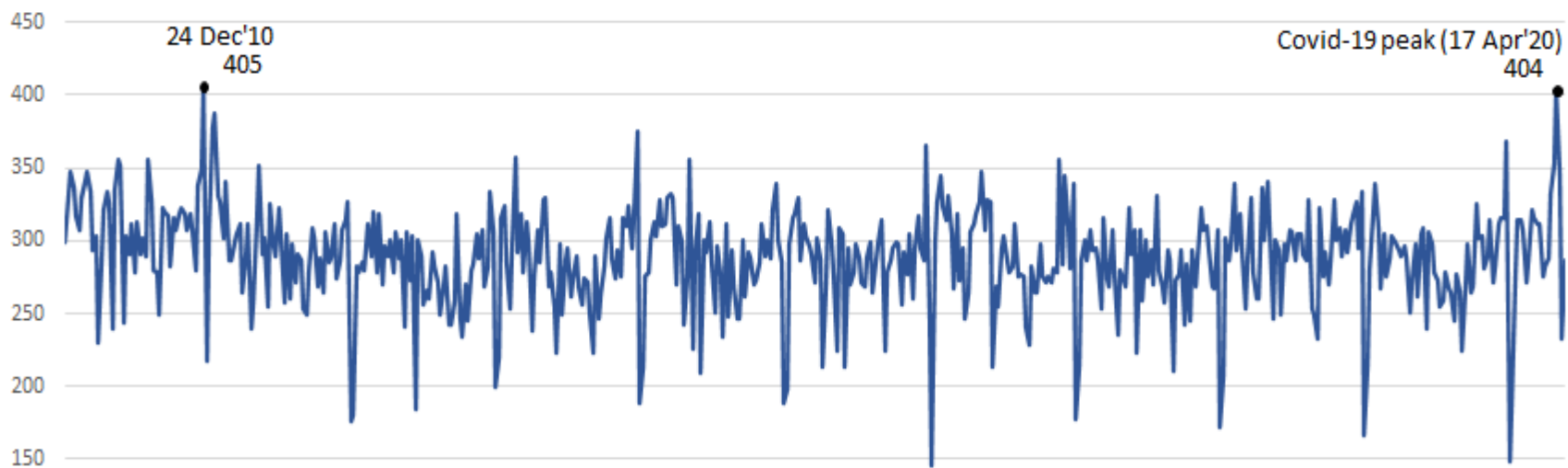

100

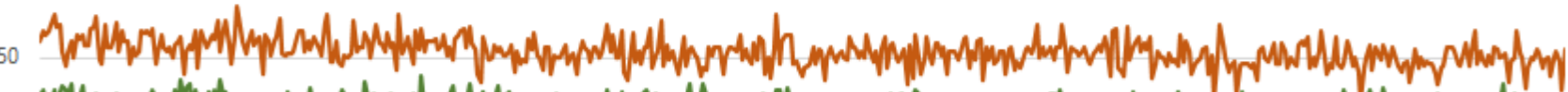

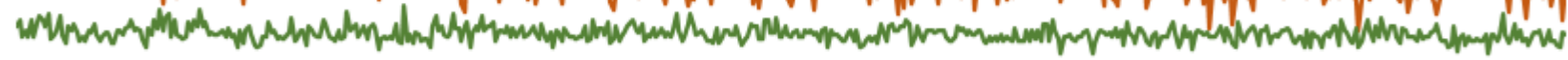

я Я 9 9 Я

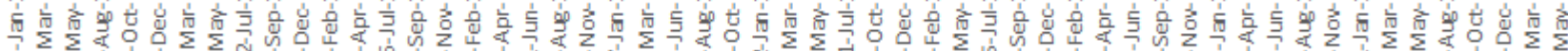

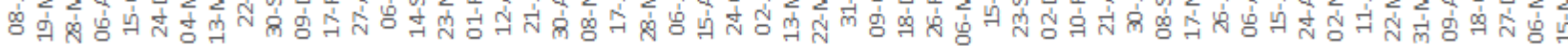


Figure 2b: Weekly deaths in England and Wales for a further four age categories from 8 January 2010-15

May 2020

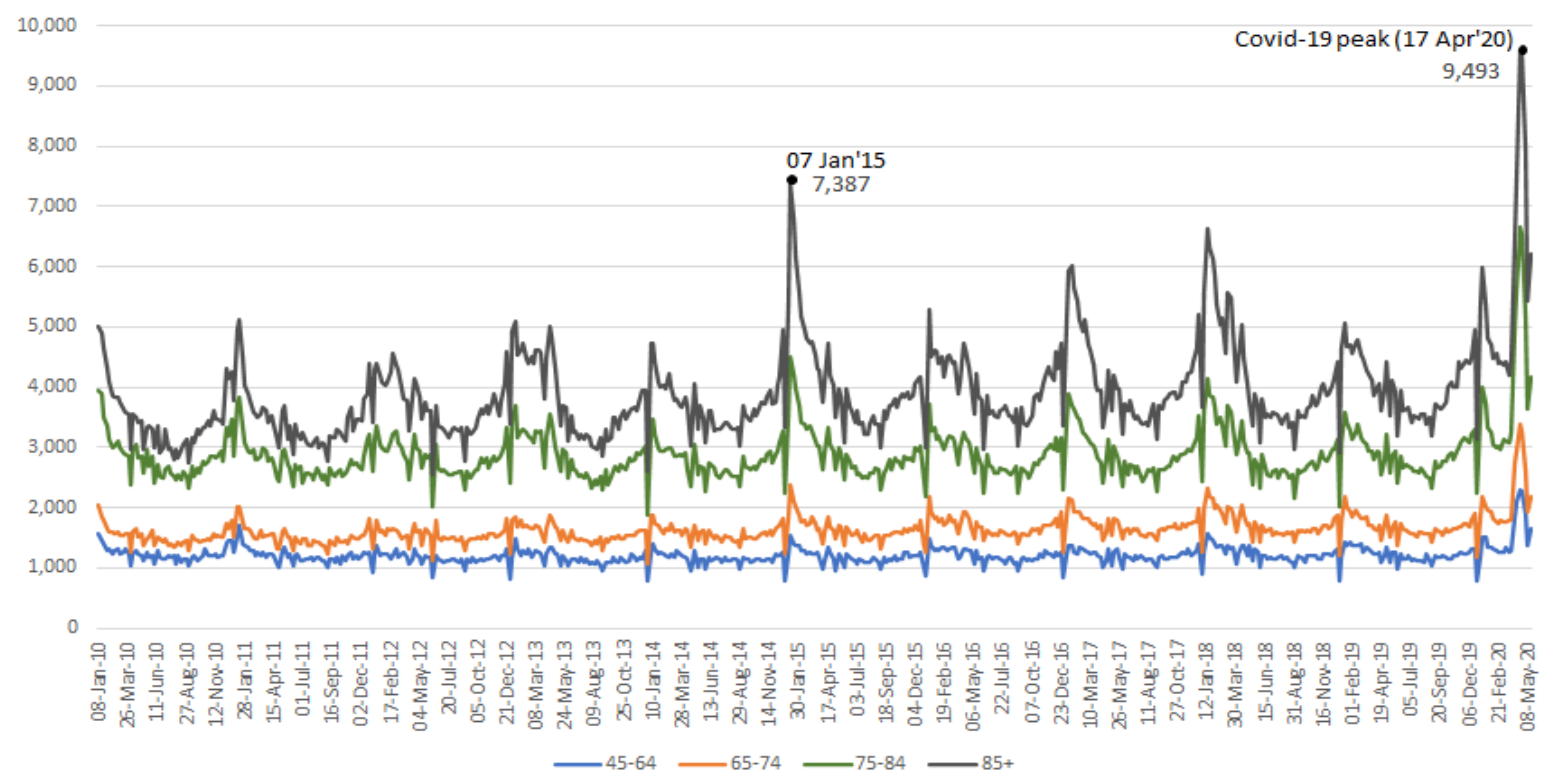

Figure 3: Difference for England and Wales between peak monthly mortality in a year and the long-term average monthly mortality (January 1998-April 2020)

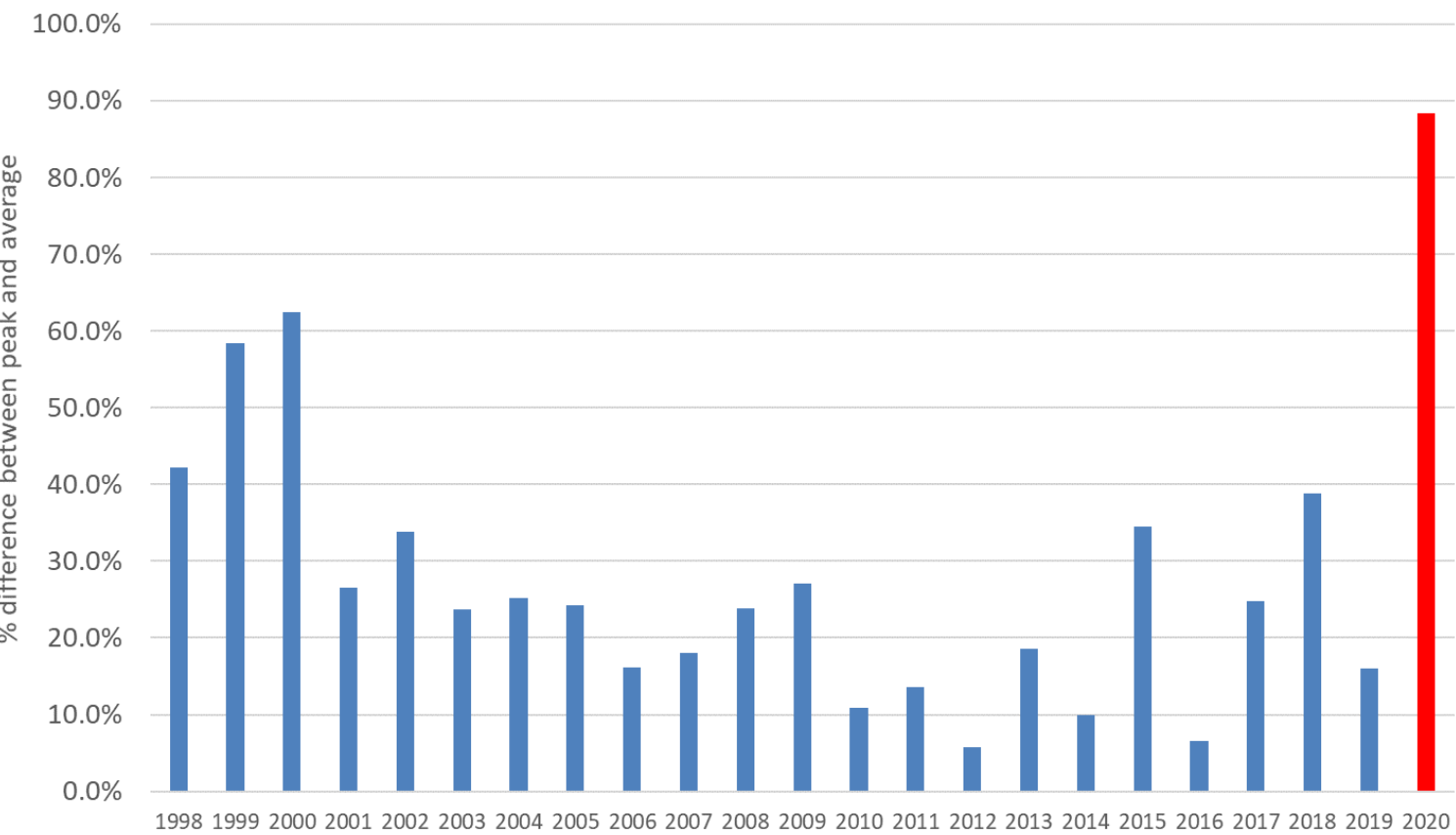


Figure 4: Peak monthly mortality in a year for England and Wales as a percentage of April 2020

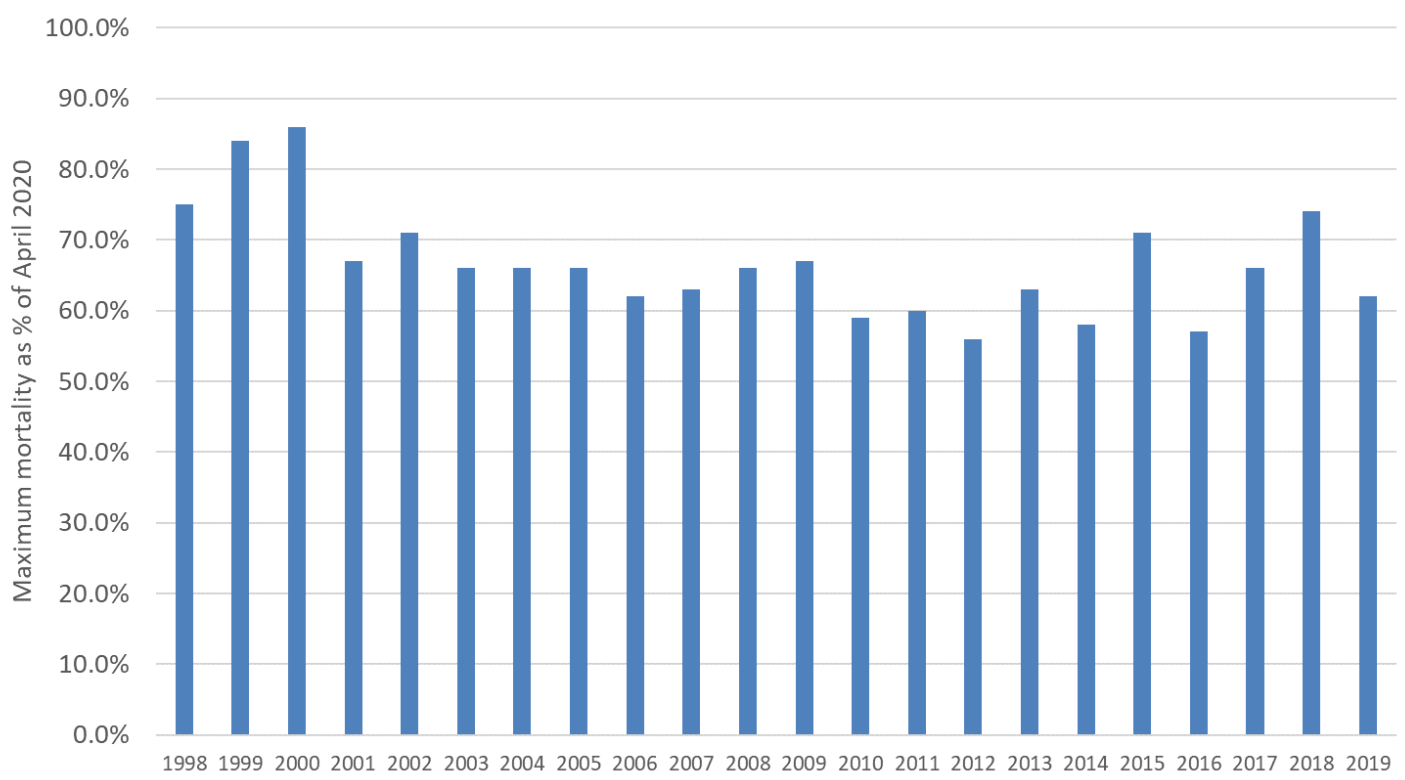

Figure 5: A\&E attendance and emergency admissions in England

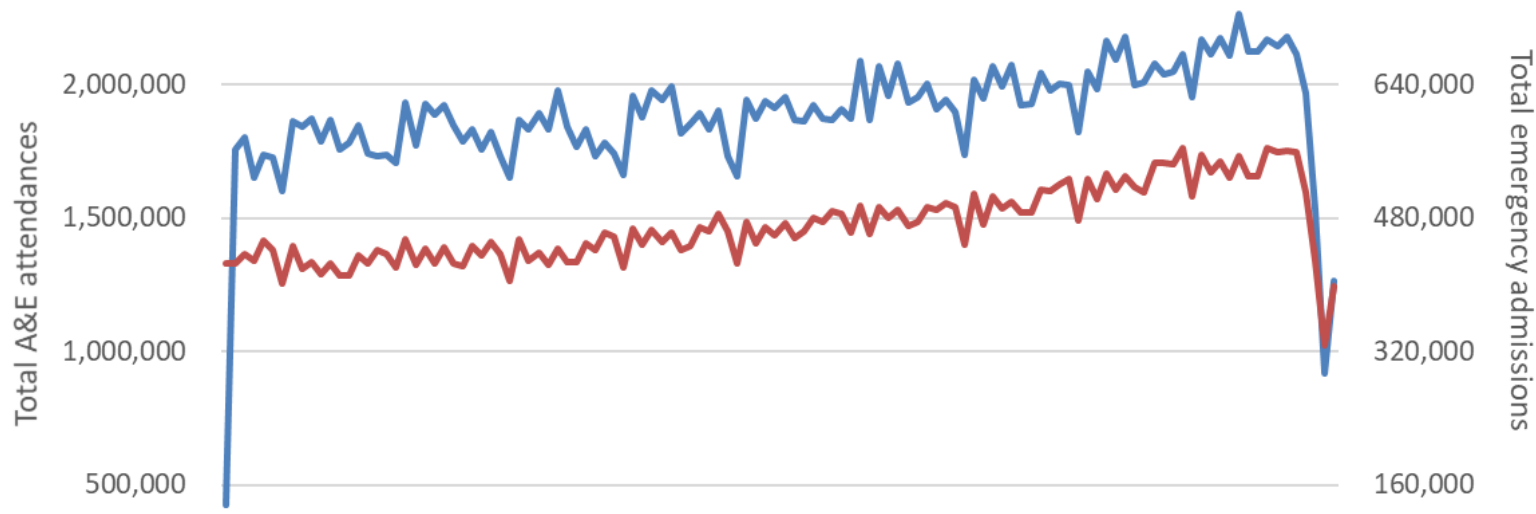

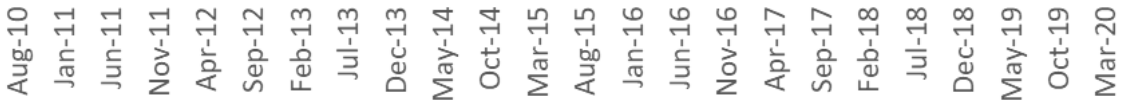

-Total Attendances —Total Emergency Admissions 
Figure 6: Excess deaths due to COVID from the Base 1 and COVID IdentV1 models

Total Deaths

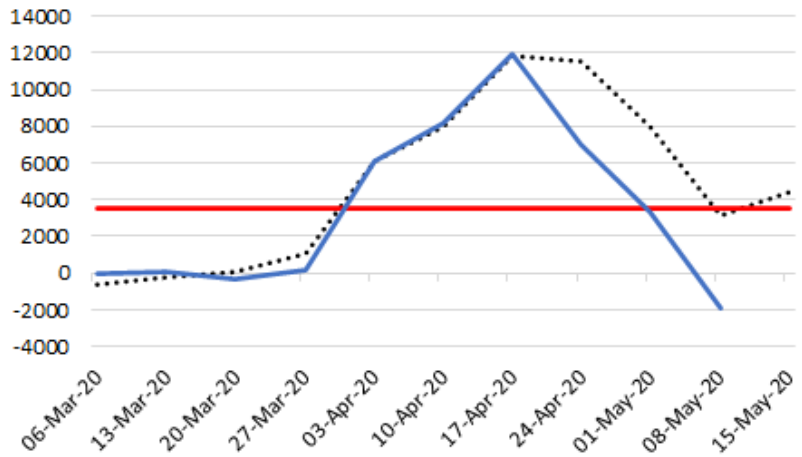

45-64

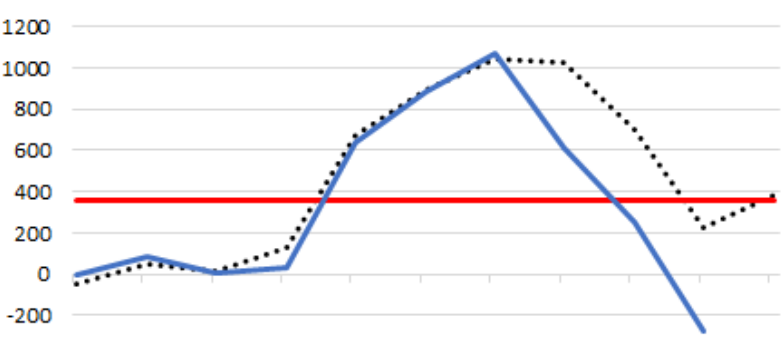

$-400$

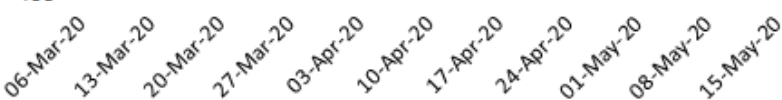

75-84

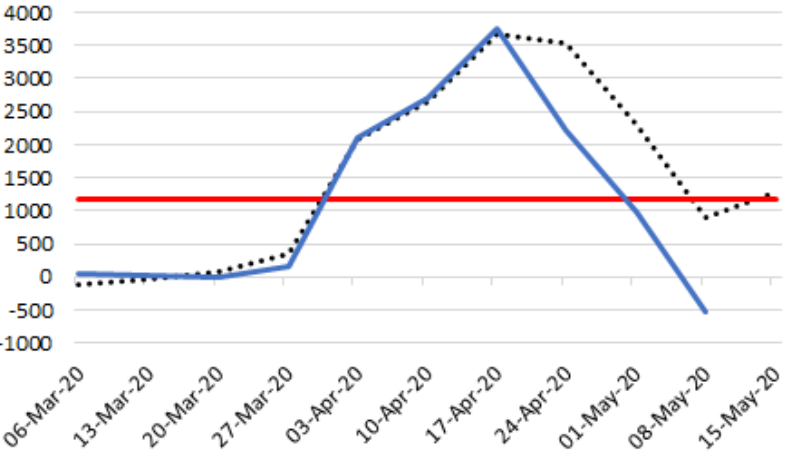

\section{Male Deaths}

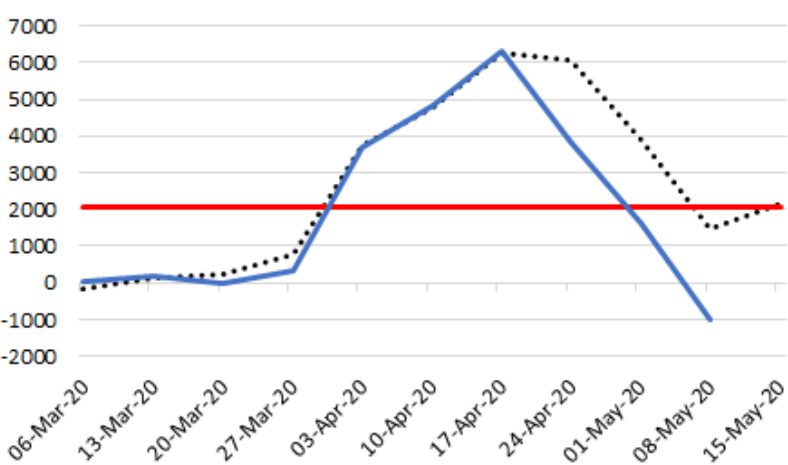

— Average weekly excess deaths (Base 1 COVID4Week)
$15-44$

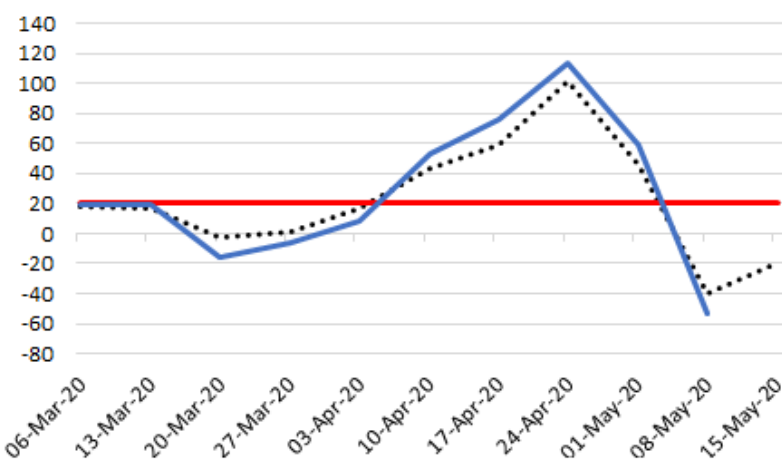

$65-74$

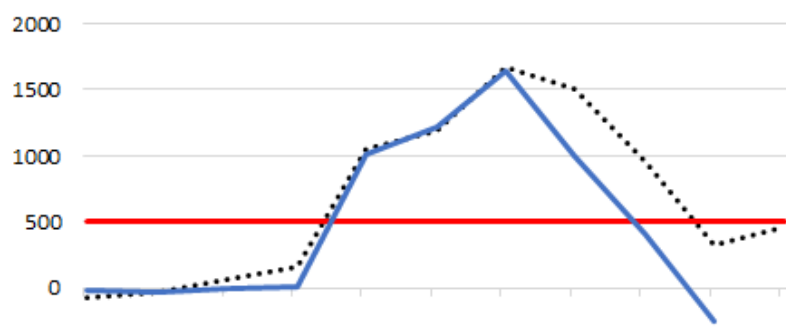

$-500$

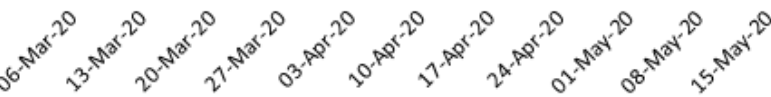

$85+$

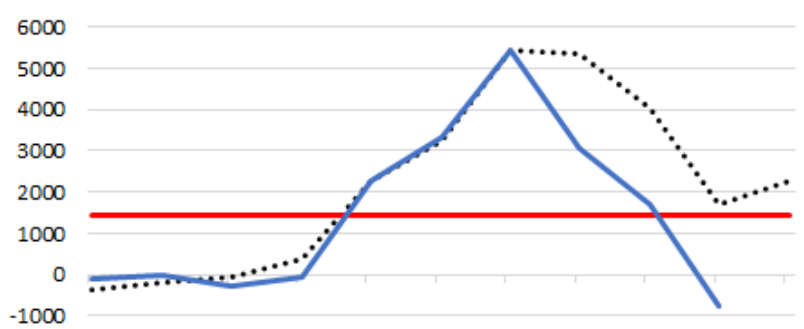

$-2000$

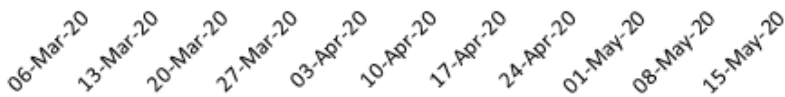

\section{Female Deaths}

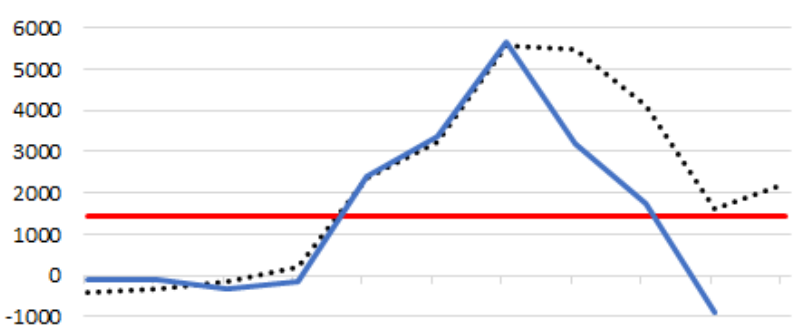

$-2000$

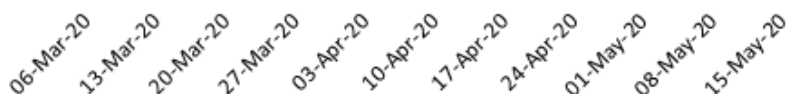

Weekly excess deaths (COVID IdentV1)

$\cdots \cdots 5$ year average excess deaths 
Figure 7: Excess deaths due to COVID from the Base 2 and COVID IdentV2 models

Total Deaths

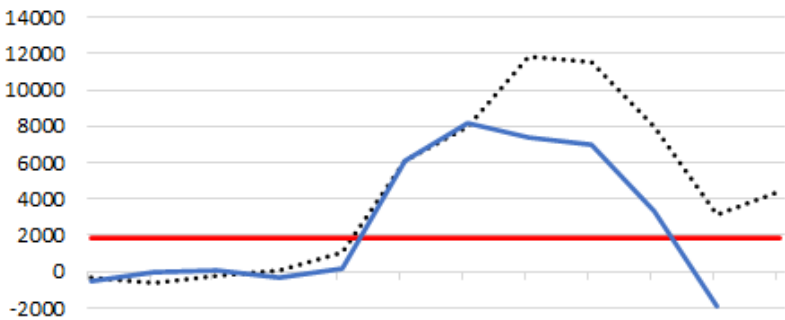

$-4000$

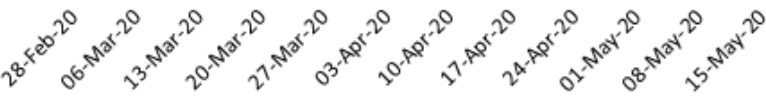

45-64

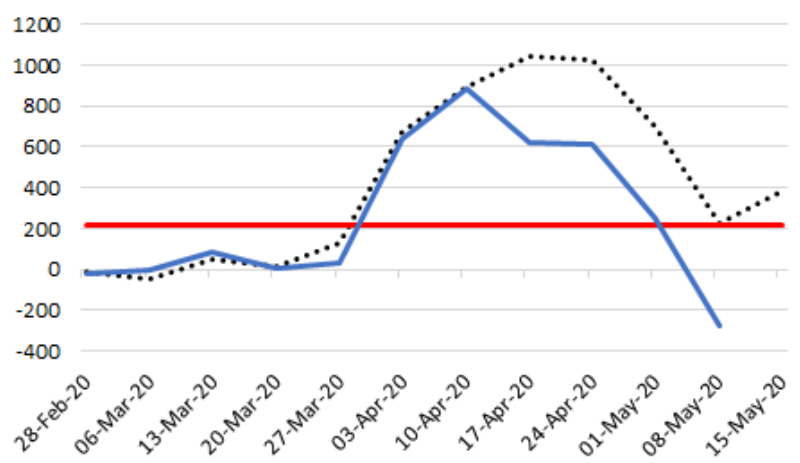

75-84

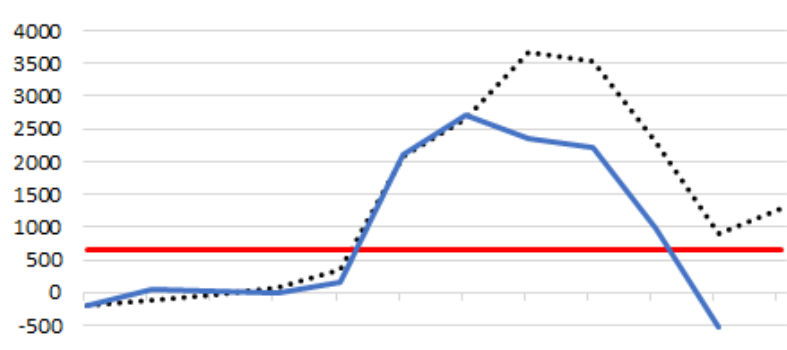

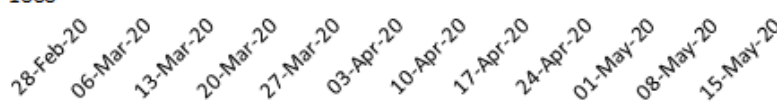

Male Deaths

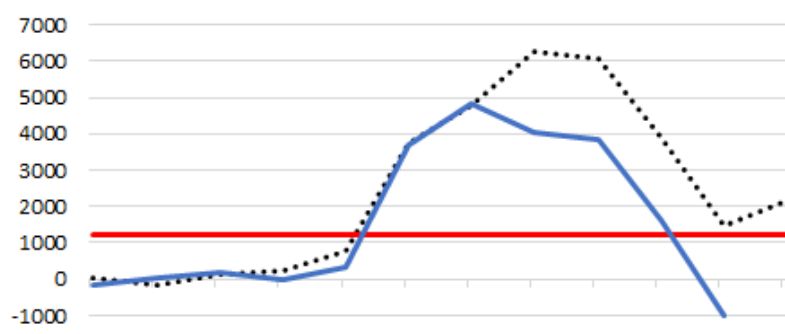

$-2000$

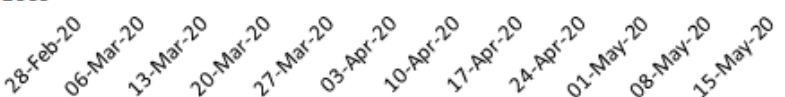

$15-44$

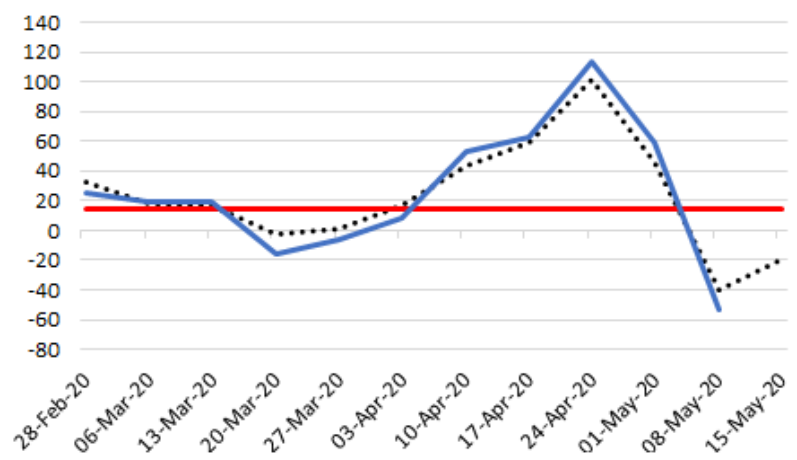

$65-74$

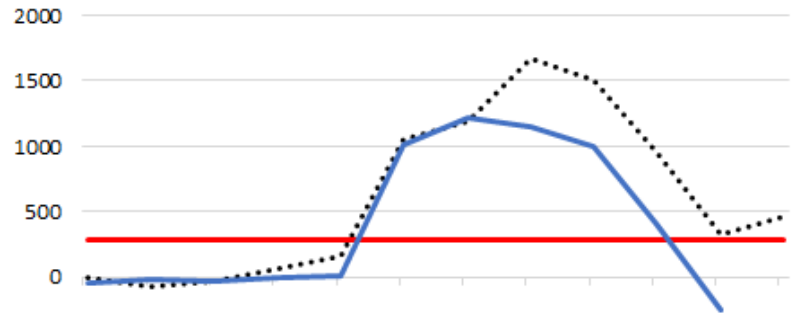

$-500$

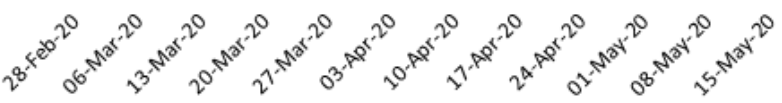

$85+$
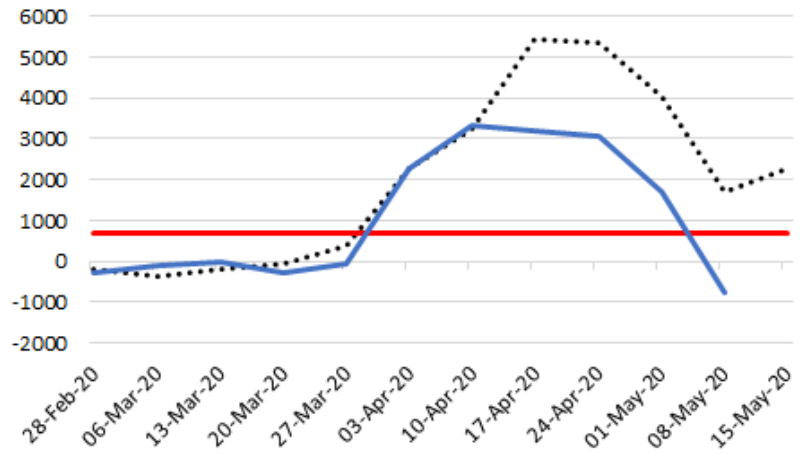

Female Deaths

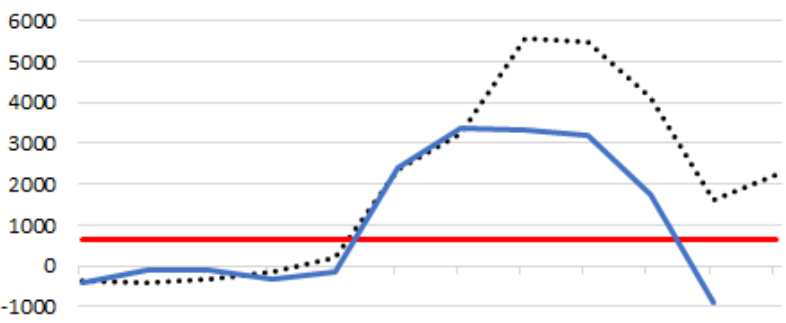

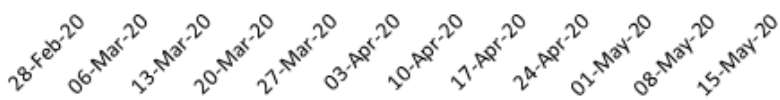


Figure 8: Change in deaths due to the lockdown from the Base 1 and LockD IdentV1 models

Total Deaths

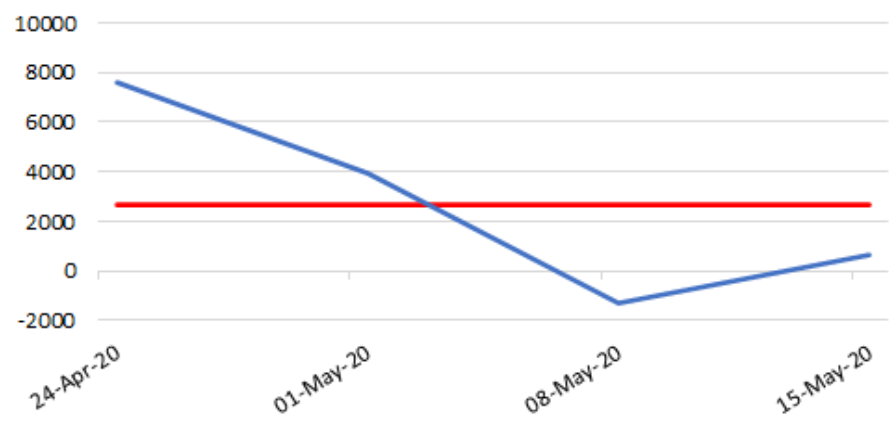

45-64

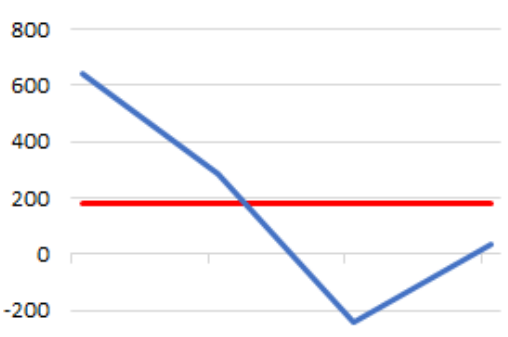

$-400$

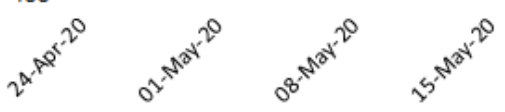

$85+$

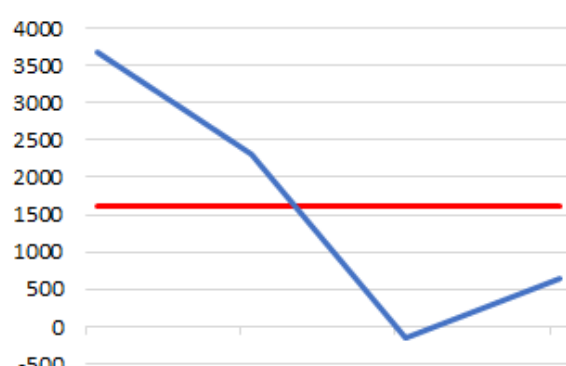

$-500$

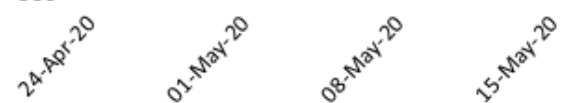

65-74

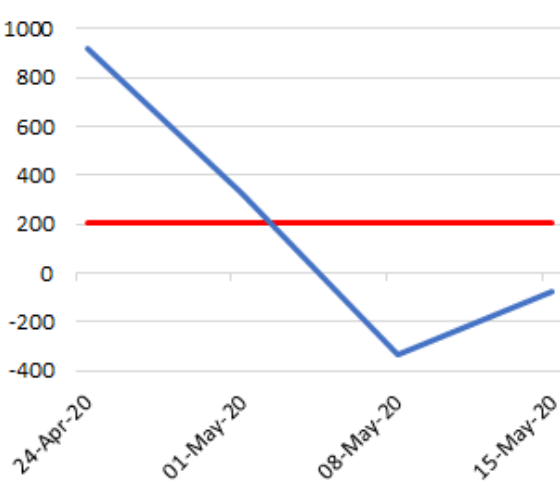

Male Deaths

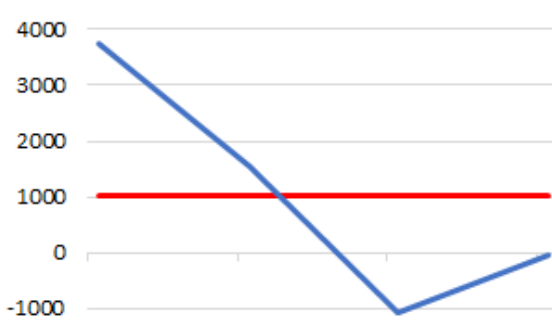

$-2000$

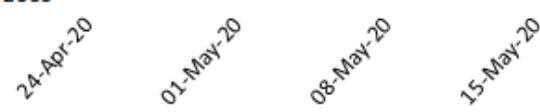

75-84

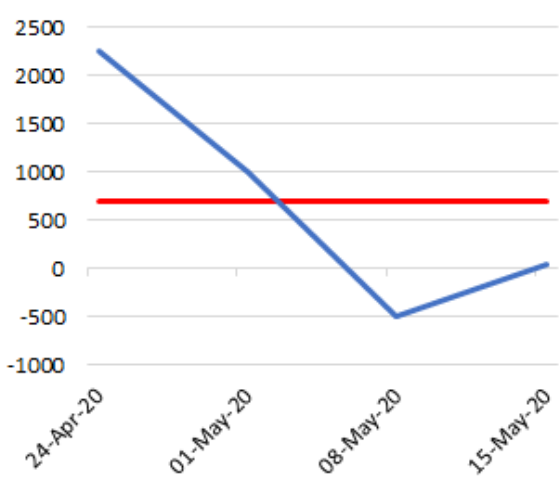

Female Deaths

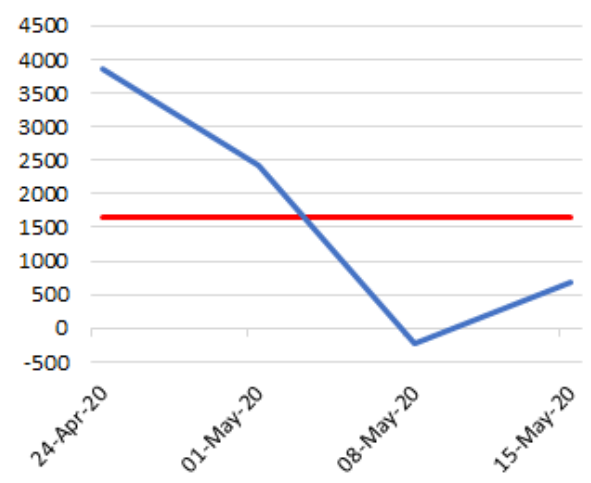

_ Average weekly deaths (Base 1 LockD4Week) _ Weekly deaths (LockD IdentV1) 
Figure 9: Change in deaths due to the lockdown from the Base 2 and LockD IdentV2 models

Total Deaths

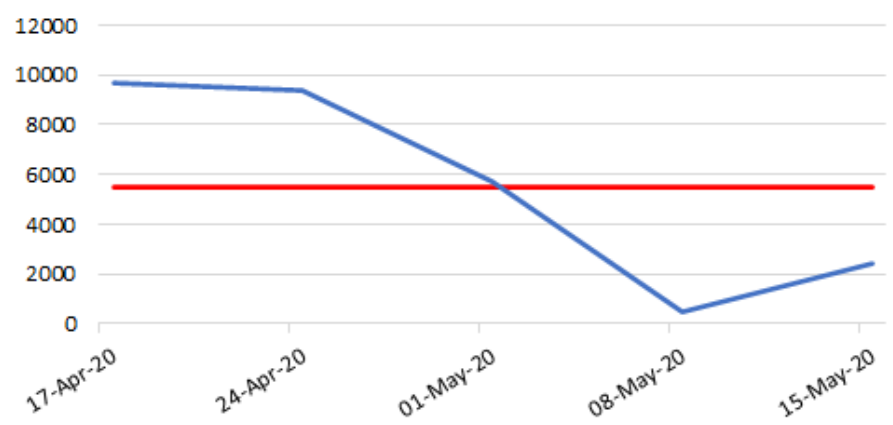

45-64

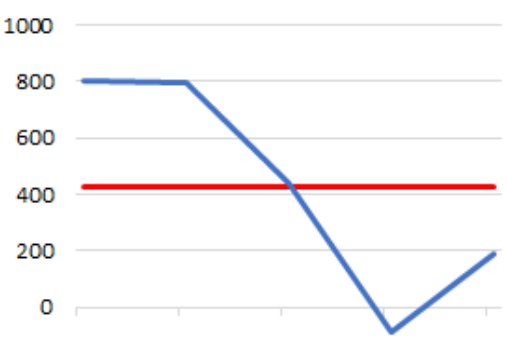

$-200$

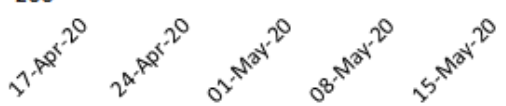

$85+$

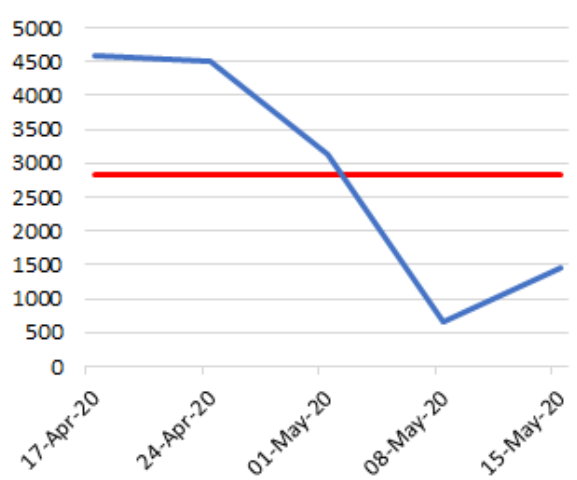

65-74

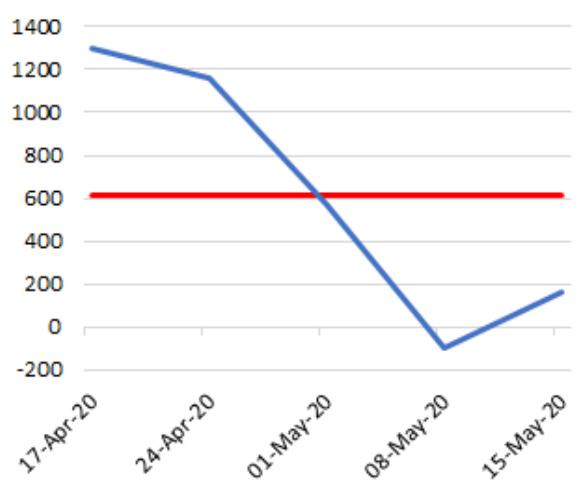

Male Deaths

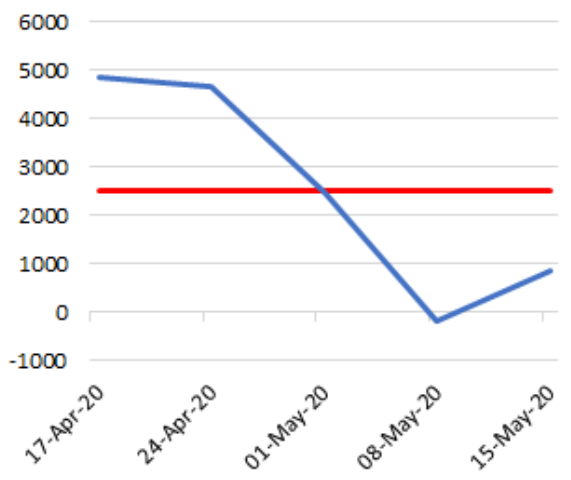

75-84

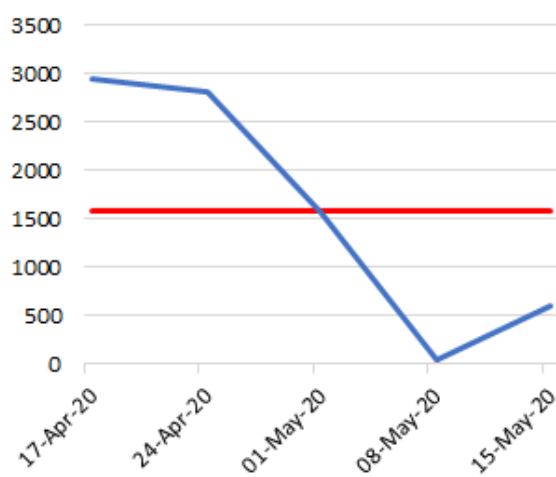

Female Deaths

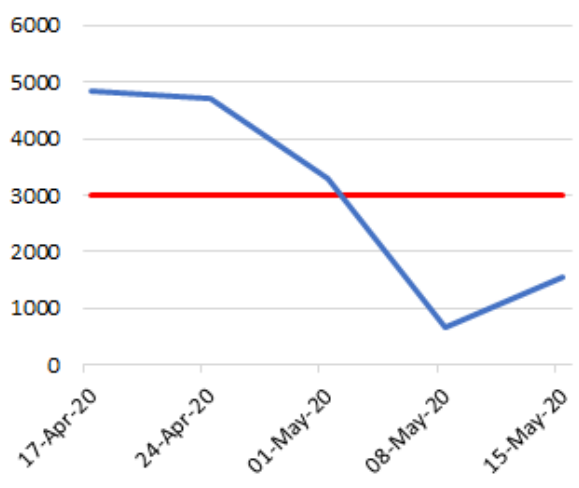

— Average weekly deaths (Base 2 LockD3Week) — Weekly deaths (LockD IdentV2) 
Table 1: COVID associated deaths in England and Wales up to the week ending 29 May 2020

\begin{tabular}{cc}
\hline \hline Age group & Proportion of COVID deaths accounted for \\
\hline$<10$ & $0.0 \%$ \\
$10-19$ & $0.0 \%$ \\
$20-29$ & $0.2 \%$ \\
$30-39$ & $0.4 \%$ \\
$40-49$ & $1.4 \%$ \\
$50-59$ & $4.6 \%$ \\
$60-69$ & $9.8 \%$ \\
$70-79$ & $22.6 \%$ \\
$80-89$ & $39.4 \%$ \\
$90+$ & $21.6 \%$ \\
\hline Total & $100 \%$ \\
\hline
\end{tabular}

Note: The COVID associated measure is used here purely for the purpose of indicting the distribution of deaths by age. As discussed above, it is unlikely to provide a reliable measure of the number of deaths arising from the pandemic.

Table 2: Data sources and summary statistics

\begin{tabular}{cccccc}
\hline \hline Variable & Source & Mean & St. Dev. & Min & Max \\
\hline Total Deaths & ONS & 10005.9 & 1605.3 & 6606.0 & 22351.0 \\
Deaths(<1) & ONS & 54.3 & 9.5 & 22.0 & 85.0 \\
Deaths(1-14) & ONS & 19.4 & 5.0 & 7.0 & 38.0 \\
Deaths(15-44) & ONS & 288.4 & 35.1 & 146.0 & 405.0 \\
Deaths(45-64) & ONS & 1207.9 & 145.3 & 773.0 & 2294.0 \\
Deaths(65-74) & ONS & 1634.2 & 223.9 & 1060.0 & 3380.0 \\
Deaths(75-84) & ONS & 2886.8 & 459.0 & 1890.0 & 6657.0 \\
Deaths(85+) & ONS & 3912.8 & 804.5 & 2548.0 & 9601.0 \\
Male Deaths & ONS & 4896.9 & 789.7 & 3203.0 & 11445.0 \\
Female Deaths & ONS & 5106.8 & 835.3 & 3385.0 & 10906.0 \\
Share90+ & ONS & $0.9 \%$ & $0.1 \%$ & $0.7 \%$ & $1.0 \%$ \\
PopDen & ONS & 382.2 & 8.2 & 369.0 & 394.0 \\
Temp & Met Office & 9.9 & 4.6 & -0.5 & 18.7 \\
FTSE All & Datastream & 3497.3 & 458.4 & 2607.9 & 4252.2 \\
\hline \hline
\end{tabular}


Table 3: Total, male and female Base 1 models with COVID4Week and LockD4Week dummy variables

\begin{tabular}{|c|c|c|c|}
\hline Variable & Total Deaths & Male Deaths & Female Deaths \\
\hline FTSE All & $-0.619 * * *$ & $-0.268 * * *$ & $-0.340 * * *$ \\
\hline Temp & $-234.76 * * *$ & $-90.37 * * *$ & $-145.04 * * *$ \\
\hline Temp ${ }^{2}$ & $3.78 * * *$ & $1.20 * * *$ & $2.62 * * *$ \\
\hline Winter & $424.07 * * *$ & $178.74 * * *$ & $245.73 * * *$ \\
\hline Summer & $50.69 * *$ & 13.58 & $36.29 * *$ \\
\hline Share90+ & 491.62 & $-680.73 * * *$ & $1154.45^{* * *}$ \\
\hline PopDen & $74.93 * * *$ & $49.44 * * *$ & $25.53 * * *$ \\
\hline COVID4Week & $3497.14 * * *$ & $2072.52 * * *$ & $1427.82 * * *$ \\
\hline LockD4Week & $2693.21 * * *$ & $1029.21 * * *$ & $1662.40 * * *$ \\
\hline Constant & $-15256.90 * * *$ & $-11800.88 * * *$ & $-3469.12 * * *$ \\
\hline Log-likelihood & -27718.29 & -14558.91 & -16348.69 \\
\hline
\end{tabular}


Table 4: Age category Base 1 models with COVID4Week and LockD4Week dummy variables

\begin{tabular}{|c|c|c|c|c|c|c|c|c|}
\hline \multirow[b]{2}{*}{ Variable } & \multirow{2}{*}{ Total Deaths } & \multicolumn{7}{|c|}{ Deaths by Age Category } \\
\hline & & $<1$ & $1-14$ & $15-44$ & $45-64$ & $65-74$ & $75-84$ & $85+$ \\
\hline FTSE All & $-0.619 * * *$ & -0.001 & 0.001 & 0.004 & $-0.065 * * *$ & $-0.088 * * *$ & $-0.193 * * *$ & $-0.273 * * *$ \\
\hline Temp & $-234.76 * * *$ & $0.97^{*}$ & -0.45 & -0.89 & $-18.22 * * *$ & $-20.09 * * *$ & $-68.68 * * *$ & $-126.90 * * *$ \\
\hline Temp ${ }^{2}$ & $3.78 * * *$ & -0.04 & 0.01 & -0.05 & $0.39 * * *$ & 0.18 & $1.10 * * *$ & $2.19 * * *$ \\
\hline Winter & $424.07 * * *$ & 1.04 & -0.04 & -3.32 & $22.07 * * *$ & $58.17 * * *$ & $107.24 * * *$ & $239.13 * * *$ \\
\hline Summer & $50.69 * *$ & -0.5 & -0.96 & 3.23 & 1.02 & 10.13 & $19.88^{*}$ & 16.99 \\
\hline Share90+ & 491.62 & $-72.39 * * *$ & 5.21 & $-381.46 * * *$ & $-1171.59 * * *$ & $-336.39 * *$ & -23.57 & $2409.89 * * *$ \\
\hline PopDen & $74.93 * * *$ & 0.21 & -0.21 & $2.71 * * *$ & $15.17^{* * *}$ & $17.23 * * *$ & $16.59 * * *$ & $23.87 * * *$ \\
\hline COVID4Week & $3497.14 * * *$ & -0.99 & -1.25 & $20.36 * *$ & $360.68 * * *$ & $508.77 * * *$ & $1182.00 * * *$ & $1428.59 * * *$ \\
\hline LockD4Week & $2693.21^{* * *}$ & -3.42 & -0.17 & 18.47 & $178.91 * * *$ & $205.23 * * *$ & $690.64 * * *$ & $1600.74^{* * *}$ \\
\hline Constant & $-15256.90 * * *$ & 38.23 & $94.31^{* *}$ & $-407.96 * * *$ & $-3203.37 * * *$ & $-4197.86 * * *$ & $-2268.57 * * *$ & $-5499.82 * * *$ \\
\hline Log-likelihood & -27718.29 & -1947.23 & -1598.25 & -3116.86 & -4957.20 & -5969.43 & -9885.67 & -16093.56 \\
\hline
\end{tabular}

Note: ${ }^{*}, * *$ and $* * *$ denote significant at the $5 \%, 1 \%$ and $0.1 \%$ levels, respectively. 
Table 5: Total, male and female COVID IdentV1 models with the LockD4Week dummy variable and 10 weekly COVID identifier variables

\begin{tabular}{|c|c|c|c|}
\hline Variable & Total Deaths & Male Deaths & Female Deaths \\
\hline FTSE All & $-0.121 * * *$ & -0.015 & $-0.104 * * *$ \\
\hline Temp & $-252.63 * * *$ & $-99.28 * * *$ & $-153.87 * * *$ \\
\hline Temp ${ }^{2}$ & $4.16 * * *$ & $1.38 * * *$ & $2.81^{* * *}$ \\
\hline Winter & $384.26 * * *$ & $158.91 * * *$ & $226.01 * * *$ \\
\hline Summer & $92.56 * * *$ & $35.12^{* *}$ & $56.49 * * *$ \\
\hline Share90+ & -482.24 & $-1182.87^{* * *}$ & $685.33^{* * *}$ \\
\hline PopDen & $58.40^{* * *}$ & $40.95^{* * *}$ & $17.54^{* * *}$ \\
\hline COVID6Mar & -33.42 & 58.07 & -92.75 \\
\hline COVID13Mar & 78.94 & $168.61^{*}$ & -89.7 \\
\hline COVID20Mar & $-311.75 * *$ & -5.48 & $-302.96 * * *$ \\
\hline COVID27Mar & 149.67 & $323.19 * * *$ & $-169.52 *$ \\
\hline COVID3Apr & $6056.51 * * *$ & $3672.39 * * *$ & $2387.87 * * *$ \\
\hline COVID10Apr & $8165.75^{* * *}$ & $4823.92 * * *$ & $3345.98 * * *$ \\
\hline COVID17Apr & $11981.26 * * *$ & $6318.48 * * *$ & $5667.33^{* * *}$ \\
\hline COVID24Apr & $7011.00 * * *$ & $3831.28 * * *$ & $3197.70 * * *$ \\
\hline CoVID1May & $3368.54 * * *$ & $1629.56 * * *$ & $1756.20 * * *$ \\
\hline COVID8May & $-1923.00 * * *$ & $-1017.88 * * *$ & $-887.99 * * *$ \\
\hline LockD4Week & $4623.50 * * *$ & $2271.10 * * *$ & $2338.81 * * *$ \\
\hline Constant & $-9664.79 * * *$ & $-8932.57 * * *$ & -763.35 \\
\hline Log-likelihood & -21625.53 & -11116.41 & -13647.02 \\
\hline
\end{tabular}

Note: ${ }^{*},{ }^{* *}$ and ${ }^{* * *}$ denote significant at the $5 \%, 1 \%$ and $0.1 \%$ levels, respectively. 
Table 6: Age category COVID IdentVI models with the LockD4Week dummy variable and 10 weekly COVID identifier variables

\begin{tabular}{|c|c|c|c|c|c|c|c|c|}
\hline \multirow[b]{2}{*}{ Variable } & \multirow{2}{*}{ Total Deaths } & \multicolumn{7}{|c|}{ Deaths by Age Category } \\
\hline & & $<1$ & $1-14$ & $15-44$ & $45-64$ & $65-74$ & $75-84$ & $85+$ \\
\hline FTSE All & $-0.121 * * *$ & -0.002 & 0.001 & 0.008 & $-0.016^{*}$ & -0.015 & $-0.041 * *$ & $-0.066 * * *$ \\
\hline Temp & $-252.63 * * *$ & $0.97^{*}$ & -0.46 & -1.03 & $-19.96 * * *$ & $-22.57 * * *$ & $-74.41 * * *$ & $-134.63 * * *$ \\
\hline Temp ${ }^{2}$ & $4.16 * * *$ & -0.04 & 0.01 & -0.05 & $0.42 * * *$ & 0.23 & $1.22 * * *$ & $2.36 * * *$ \\
\hline Winter & $384.26 * * *$ & 1.11 & -0.08 & -3.37 & $18.11 * * *$ & $52.52 * * *$ & $94.64 * * *$ & $222.03 * * *$ \\
\hline Summer & $92.56 * * *$ & -0.66 & -0.92 & 3.06 & 5.4 & $16.71^{*}$ & $32.86 * * *$ & $34.03 * *$ \\
\hline Share90+ & -482.24 & $-71.17^{* * *}$ & 5.64 & $-388.37 * * *$ & $-1268.08 * * *$ & $-478.71 * * *$ & $-325.25^{*}$ & $1995.10 * * *$ \\
\hline PopDen & $58.40 * * *$ & 0.23 & -0.2 & $2.59 * * *$ & $13.55^{* * *}$ & $14.76^{* * *}$ & $11.46 * * *$ & $16.87^{* * *}$ \\
\hline COVID6Mar & -33.42 & 7.15 & 1.26 & 19.08 & -5.68 & -16.4 & 52.27 & -89.41 \\
\hline COVID13Mar & 78.94 & 4 & 3.31 & 18.8 & $80.75^{*}$ & -33.8 & 29.36 & -21.73 \\
\hline COVID20Mar & $-311.75 * *$ & -5.21 & -6.61 & -16.15 & 2.5 & -8.8 & -13.24 & $-260.79 * * *$ \\
\hline COVID27Mar & 149.67 & -0.65 & -5.46 & -5.99 & 34.84 & 12.04 & $155.14^{* *}$ & -39.57 \\
\hline COVID3Apr & $6056.51^{* * *}$ & -0.24 & 3.58 & 8.25 & $637.84 * * *$ & $1007.86 * * *$ & $2106.28 * * *$ & $2287.03^{* * *}$ \\
\hline COVID10Apr & $8165.75 * * *$ & $-13.49 *$ & -3.33 & $53.49 * *$ & $886.18 * * *$ & $1217.49 * * *$ & $2707.65^{* * *}$ & $3310.30 * * *$ \\
\hline COVID17Apr & $11981.26 * * *$ & -0.73 & -2.24 & $75.70 * * *$ & $1066.55^{* * *}$ & $1649.15^{* * *}$ & $3745.10 * * *$ & $5438.72 * * *$ \\
\hline COVID24Apr & 7011.00*** & -2.22 & -7.24 & $113.26 * * *$ & $611.53 * * *$ & $1004.35 * * *$ & $2223.33 * * *$ & $3067.93 * * *$ \\
\hline CoVID1May & $3368.54 * * *$ & -8.15 & -7.95 & $58.72 *$ & $252.45 * * *$ & $411.62 * * *$ & $971.15^{* * *}$ & $1689.78 * * *$ \\
\hline COVID8May & $-1923.00 * * *$ & $-28.09 * *$ & 1.03 & $-53.56^{*}$ & $-273.95 * * *$ & $-253.84 * * *$ & $-542.35 * * *$ & $-772.80 * * *$ \\
\hline LockD4Week & $4623.50 * * *$ & 4.57 & 1.97 & 12.99 & $445.00 * * *$ & $503.67 * * *$ & $1380.20 * * *$ & $2266.73 * * *$ \\
\hline Constant & $-9664.79 * * *$ & 31.96 & $92.90 * *$ & $-369.64 * *$ & $-2657.24 * * *$ & $-3365.08 * * *$ & -533.57 & $-3124.59 * * *$ \\
\hline Log-likelihood & -21625.53 & -1938.71 & -1593.04 & -3082.21 & -4473.70 & -5167.83 & -7848.40 & -13200.47 \\
\hline
\end{tabular}

Note: $*, * *$ and $* * *$ denote significant at the $5 \%, 1 \%$ and $0.1 \%$ levels, respectively. 
Table 7: Total, male and female LockD IdentVI models with the COVID4Week dummy variable and 4 weekly lockdown identifier variables

\begin{tabular}{llll}
\hline \hline & & & \\
Variable & Total Deaths & Male Deaths & Female Deaths \\
\cline { 2 - 4 } FTSE All & & & \\
Temp & $-0.589 * * *$ & $-0.256 * * *$ & $-0.330 * * *$ \\
Temp & $-236.29 * * *$ & $-91.23^{* * *}$ & $-145.71^{* * *}$ \\
Winter & $3.99 * * *$ & $1.31 * * *$ & $2.71 * * *$ \\
Summer & $434.22 * * *$ & $184.24 * * *$ & $250.37 * * *$ \\
Share90+ & 29.13 & 1.72 & $26.59 *$ \\
PopDen & 448.06 & $-703.84 * * *$ & $1133.97 * * *$ \\
COVID4Week & $74.05 * * *$ & $48.97 * * *$ & $25.12 * * *$ \\
LockD24Apr & $7517.76 * * *$ & $2083.58 * * *$ & $1437.38 * * *$ \\
LockD1May & $3966.61 * * *$ & $3752.68 * * *$ & $3848.72 * * *$ \\
LockD8May & $-1307.74 * * *$ & $-1082.38 * * *$ & $-223.46 * *$ \\
LockD15May & $642.28 * * *$ & -50.61 & $677.57 * * *$ \\
Constant & $-14970.27 * * *$ & $-11648.21 * * *$ & $-3335.22 * * *$ \\
\hline Log-likelihood & -26371.29 & -13780.34 & -15766.85 \\
\hline \hline
\end{tabular}

Note: $*, * *$ and $* * *$ denote significant at the $5 \%, 1 \%$ and $0.1 \%$ levels, respectively. 
Table 8: Age category LockD IdentVI models with the COVID4Week dummy variable and 4 weekly lockdown identifier variables

\begin{tabular}{|c|c|c|c|c|c|c|c|c|}
\hline \multirow[b]{2}{*}{ Variable } & \multirow{2}{*}{ Total Deaths } & \multicolumn{7}{|c|}{ Deaths by Age Category } \\
\hline & & $<1$ & $1-14$ & $15-44$ & $45-64$ & $65-74$ & $75-84$ & $85+$ \\
\hline FTSE All & $-0.589 * * *$ & -0.001 & 0.001 & 0.005 & $-0.063 * * *$ & $-0.084 * * *$ & $-0.186 * * *$ & $-0.263 * * *$ \\
\hline Temp & $-236.29 * * *$ & $0.96 *$ & -0.45 & -0.94 & $-18.40 * * *$ & $-20.35 * * *$ & $-69.17 * * *$ & $-127.43 * * *$ \\
\hline Temp ${ }^{2}$ & $3.99 * * *$ & -0.04 & 0.01 & -0.05 & $0.41 * * *$ & 0.22 & $1.17^{* * *}$ & $2.26 * * *$ \\
\hline Winter & $434.22 * * *$ & 1.07 & -0.06 & -3.06 & $23.13 * * *$ & $59.78 * * *$ & $110.46 * * *$ & $243.05 * * *$ \\
\hline Summer & 29.13 & -0.58 & -0.93 & 2.63 & -1.38 & 6.59 & 13.02 & 9.02 \\
\hline Share90+ & 448.06 & $-72.36 * * *$ & 5.31 & $-382.50 * * *$ & $-1175.76 * * *$ & $-342.91 * *$ & -37.16 & $2392.01 * * *$ \\
\hline PopDen & $74.05^{* * *}$ & 0.21 & -0.2 & $2.69 * * *$ & $15.08 * * *$ & $17.10 * * *$ & $16.32 * * *$ & $23.52 * * *$ \\
\hline COVID4Week & $3517.76 * * *$ & -0.96 & -1.29 & $20.85^{* *}$ & $362.71 * * *$ & $511.94 * * *$ & $1188.48 * * *$ & $1436.90 * * *$ \\
\hline LockD24Apr & $7598.56 * * *$ & 3.98 & -3.88 & $102.26 * * *$ & $642.65 * * *$ & $919.17 * * *$ & $2254.13^{* * *}$ & $3678.12 * * *$ \\
\hline LockD1May & $3966.61 * * *$ & -1.96 & -4.6 & $47.76^{*}$ & $284.41 * * *$ & $327.83^{* * *}$ & $1005.08 * * *$ & $2305.11 * * *$ \\
\hline LockD8May & $-1307.74 * * *$ & $-21.93 * * *$ & 4.38 & $-64.41 * * *$ & $-240.28 * * *$ & $-335.08 * * *$ & $-503.09 * * *$ & -150.22 \\
\hline LockD15May & $642.28 * * *$ & 6.12 & 3.34 & -10.68 & 36.34 & -77.22 & 47.66 & $633.99 * * *$ \\
\hline Constant & $-14970.27 * * *$ & 38.66 & $93.75^{* *}$ & $-400.80 * *$ & $-3174.74 * * *$ & $-4153.16 * * *$ & $-2179.36 * * *$ & $-5385.21 * * *$ \\
\hline Log-likelihood & -26371.29 & -1941.43 & -1596.08 & -3092.11 & -4840.36 & -5793.51 & -9445.02 & -15492.73 \\
\hline
\end{tabular}

Note: ${ }^{*}, *$ and ${ }^{* * *}$ denote significant at the $5 \%, 1 \%$ and $0.1 \%$ levels, respectively. 
Table 9: 5 year mean weekly excess deaths versus mean weekly excess deaths due to COVID from the COVID IdentVI models - week ending 24 April 2020-week ending 8 May 2020

\begin{tabular}{lcccc}
\hline \hline & $\begin{array}{c}\mathbf{5} \text { year mean } \\
\text { weekly excess deaths (A) }\end{array}$ & $\begin{array}{c}\text { Mean weekly excess deaths } \\
\text { due to COVID from the COVID } \\
\text { IdentV1 models (B) }\end{array}$ & $\begin{array}{c}\text { Difference } \\
\text { (B-A) }\end{array}$ & $\begin{array}{c}\% \\
\text { difference }\end{array}$ \\
\cline { 2 - 5 } Total Deaths & 7546 & 2819 & -4727 & -62.6 \\
Male & 3810 & 1481 & -2329 & -61.1 \\
Female & 3739 & 1355 & -2384 & -63.8 \\
$\mathbf{1 5 - 4 4}$ & 36 & 39 & 3 & 8.3 \\
$\mathbf{4 5 - 6 4}$ & 650 & 197 & -453 & -69.7 \\
$\mathbf{6 5 - 7 4}$ & 931 & 387 & -544 & -58.4 \\
$\mathbf{7 5 - 8 4}$ & 2253 & 884 & -1369 & -60.8 \\
$\mathbf{8 5 +}$ & 3689 & 1328 & -2361 & -64.0 \\
\hline \hline
\end{tabular}

Table 10: 5 year mean weekly excess deaths versus the mean weekly excess deaths due to COVID from the COVID IdentV2 models - week ending 17 April 2020-week ending 8 May 2020

\begin{tabular}{lcccc}
\hline \hline & $\begin{array}{c}\text { 5 year mean } \\
\text { weekly excess deaths (A) }\end{array}$ & $\begin{array}{c}\text { Mean weekly excess deaths } \\
\text { due to COVID from the COVID } \\
\text { IdentV2 models (B) }\end{array}$ & $\begin{array}{c}\text { Difference } \\
\text { (B-A) }\end{array}$ & $\begin{array}{c}\% \\
\text { difference }\end{array}$ \\
\cline { 2 - 5 } Total Deaths & 8624 & 3954 & -4670 & -54.2 \\
Male & 4427 & 2123 & -2304 & -52.0 \\
Female & 4199 & 1849 & -2350 & -56.0 \\
$\mathbf{1 5 - 4 4}$ & 42 & 45 & 3 & 7.1 \\
$\mathbf{4 5 - 6 4}$ & 749 & 303 & -446 & -59.5 \\
$\mathbf{6 5 - 7 4}$ & 1114 & 577 & -537 & -48.2 \\
$\mathbf{7 5 - 8 4}$ & 2609 & 1254 & -1355 & -51.9 \\
$\mathbf{8 5 +}$ & 4124 & 1789 & -2335 & -56.6 \\
\hline \hline
\end{tabular}




\section{Appendix}

Table A1: Total, male and female Base 2 models with COVID3Week and LockD3Week dummy variables

\begin{tabular}{|c|c|c|c|}
\hline Variable & Total Deaths & Male Deaths & Female Deaths \\
\hline FTSE All & $-0.503 * * *$ & $-0.224 * * *$ & $-0.274 * * *$ \\
\hline Temp & $-243.29 * * *$ & $-94.94 * * *$ & $-148.98 * * *$ \\
\hline Temp ${ }^{2}$ & $3.86^{* * *}$ & $1.25^{* * *}$ & $2.64^{* * *}$ \\
\hline Winter & $373.69 * * *$ & $151.20 * * *$ & $223.11 * * *$ \\
\hline Summer & $80.50 * * *$ & $27.94 *$ & $51.78 * * *$ \\
\hline Share90+ & 285.61 & $-749.86 * * *$ & $1015.70 * * *$ \\
\hline PopDen & $71.57^{* * * *}$ & $48.00 * * *$ & $23.57 * * *$ \\
\hline COVID3Week & $1837.32^{* * *}$ & $1211.15^{* * *}$ & $628.18 * * *$ \\
\hline LockD3Week & $5494.27 * * *$ & $2512.99 * * *$ & $2983.13^{* * *}$ \\
\hline Constant & $-14088.47 * * *$ & $-11298.47 * * *$ & $-2791.28 * * *$ \\
\hline Log-likelihood & -26284.24 & -13992.07 & -15485.22 \\
\hline
\end{tabular}


Table A2: Age category Base 2 models with COVID3Week and LockD3Week dummy variables

\begin{tabular}{|c|c|c|c|c|c|c|c|c|}
\hline \multirow[b]{2}{*}{ Variable } & \multirow{2}{*}{ Total Deaths } & \multicolumn{7}{|c|}{ Deaths by Age Category } \\
\hline & & $<1$ & $1-14$ & $15-44$ & $45-64$ & $65-74$ & $75-84$ & $85+$ \\
\hline FTSE All & $-0.503 * * *$ & -0.001 & 0.001 & 0.006 & $-0.057 * * *$ & $-0.074 * * *$ & $-0.167^{* * *}$ & $-0.217 * * *$ \\
\hline Temp & $-243.29 * * *$ & $0.97^{*}$ & -0.45 & -0.94 & $-19.12 * * *$ & $-21.24 * * *$ & $-71.50 * * *$ & $-130.47 * * *$ \\
\hline Temp ${ }^{2}$ & $3.86 * * *$ & -0.04 & 0.01 & -0.05 & $0.40 * * *$ & 0.19 & $1.13^{* * *}$ & $2.22 * * *$ \\
\hline Winter & $373.69 * * *$ & 1.04 & 0 & -3.56 & $16.66 * *$ & $50.74 * * *$ & $90.37 * * *$ & $219.26 * * *$ \\
\hline Summer & $80.50 * * *$ & -0.47 & -0.97 & 3.53 & 3.91 & $14.83^{*}$ & $28.71^{* *}$ & $29.30 * *$ \\
\hline Share90+ & 285.61 & $-72.52 * * *$ & 5.17 & $-383.96 * * *$ & $-1184.12 * * *$ & $-359.60 * * *$ & -71.79 & $2296.00 * * *$ \\
\hline PopDen & $71.57 * * *$ & 0.21 & -0.21 & $2.63 * * *$ & $14.89 * * *$ & $16.79 * * *$ & $15.81 * * *$ & $22.18 * * *$ \\
\hline COVID3Week & $1837.32 * * *$ & -1.1 & -0.75 & $14.28 *$ & $216.65 * * *$ & $284.57 * * *$ & $654.08 * * *$ & $670.55 * * *$ \\
\hline LockD3Week & $5494.27 * * *$ & -2.42 & -0.77 & $33.17 * *$ & $424.74 * * *$ & $611.84 * * *$ & $1579.33 * * *$ & $2842.71 * * *$ \\
\hline Constant & $-14088.47^{* * *}$ & 38.100 & $94.75 * *$ & $-382.21 * *$ & $-3106.21 * * *$ & $-4043.95 * * *$ & $-1991.18 * * *$ & $-4912.94 * * *$ \\
\hline Log-likelihood & -26284.24 & -1947.37 & -1598.32 & -3112.34 & -4886.88 & -5848.48 & -9515.08 & -15172.98 \\
\hline
\end{tabular}

Note: $*, * *$ and $* * *$ denote significant at the $5 \%, 1 \%$ and $0.1 \%$ levels, respectively. 
Table A3: Total, male and female COVID IdentV2 models with the LockD3Week dummy variable and 11 weekly COVID identifier variables

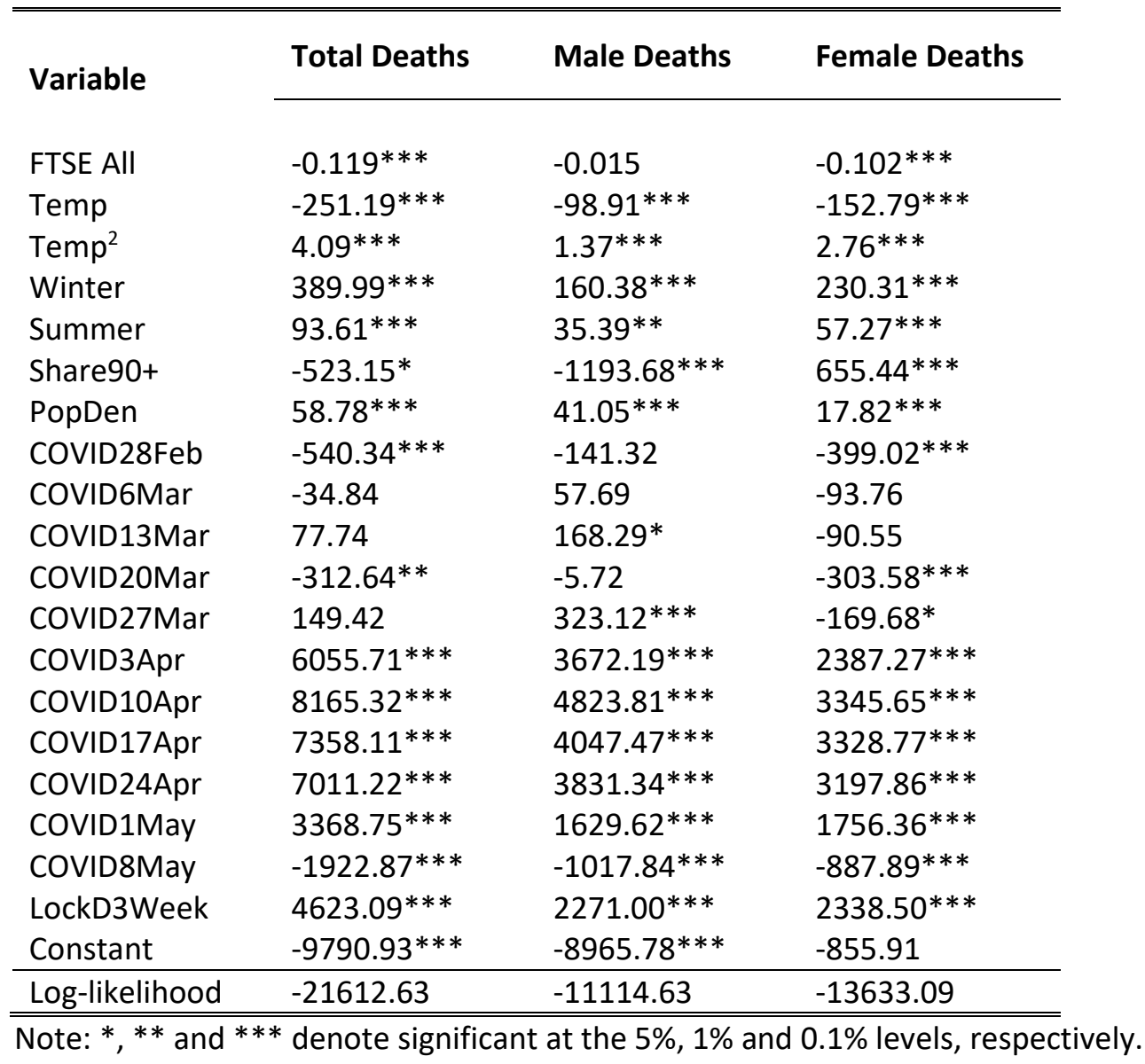


Table A4: Age category COVID IdentV2 models with the LockD3Week dummy variable and 11 weekly COVID identifier variables

\begin{tabular}{|c|c|c|c|c|c|c|c|c|}
\hline \multirow[b]{2}{*}{ Variable } & \multirow{2}{*}{ Total Deaths } & \multicolumn{7}{|c|}{ Deaths by Age Category } \\
\hline & & $<1$ & $1-14$ & $15-44$ & $45-64$ & $65-74$ & $75-84$ & $85+$ \\
\hline FTSE All & $-0.119 * * *$ & -0.002 & 0.001 & 0.007 & $-0.016^{*}$ & -0.014 & $-0.040 * *$ & $-0.065 * * *$ \\
\hline Temp & $-251.19 * * *$ & $0.97^{*}$ & -0.47 & -1.1 & $-19.90 * * *$ & $-22.44 * * *$ & $-73.83 * * *$ & $-133.91 * * *$ \\
\hline Temp ${ }^{2}$ & $4.09 * * *$ & -0.04 & 0.01 & -0.04 & $0.42 * * *$ & 0.22 & $1.20 * * *$ & $2.33^{* * *}$ \\
\hline Winter & $389.99 * * *$ & 1.12 & -0.1 & -3.65 & $18.34 * * *$ & $53.03 * * *$ & $96.93 * * *$ & $224.95 * * *$ \\
\hline Summer & $93.61 * * *$ & -0.66 & -0.92 & 3.01 & 5.45 & $16.81^{*}$ & $33.28 * * *$ & $34.54 * *$ \\
\hline Share90+ & $-523.15^{*}$ & $-71.24 * * *$ & 5.76 & $-386.20 * * *$ & $-1269.81 * * *$ & $-482.54 * * *$ & $-341.44^{*}$ & $1974.84 * * *$ \\
\hline PopDen & $58.78 * * *$ & 0.23 & -0.2 & $2.57 * * *$ & $13.57 * * *$ & $14.80 * * *$ & $11.61^{* * *}$ & $17.06 * * *$ \\
\hline COVID28Feb & $-540.34 * * *$ & -0.86 & 1.29 & 25.34 & -21.59 & -49.11 & $-210.86 * * *$ & $-281.13 * * *$ \\
\hline COVID6Mar & -34.84 & 7.15 & 1.26 & 19.15 & -5.74 & -16.54 & 51.72 & -90.11 \\
\hline CoVID13Mar & 77.74 & 4.00 & 3.31 & 18.87 & $80.70 *$ & -33.92 & 28.9 & -22.33 \\
\hline COVID20Mar & $-312.64 * *$ & -5.21 & -6.61 & -16.11 & 2.47 & -8.89 & -13.57 & $-261.24^{* * *}$ \\
\hline COVID27Mar & 149.42 & -0.65 & -5.46 & -5.98 & 34.83 & 12.02 & $155.08^{* *}$ & -39.73 \\
\hline COVID3Apr & $6055.71 * * *$ & -0.24 & 3.58 & 8.27 & $637.82 * * *$ & $1007.79 * * *$ & $2106.00 * * *$ & $2286.56 * * *$ \\
\hline COVID10Apr & $8165.32 * * *$ & $-13.49 *$ & -3.33 & $53.49 * *$ & $886.17^{* * *}$ & $1217.45^{* * *}$ & $2707.51 * * *$ & $3310.00 * * *$ \\
\hline COVID17Apr & $7358.11 * * *$ & -5.31 & -4.21 & $62.69 *$ & $621.57 * * *$ & $1145.51 * * *$ & $2365.05 * * *$ & $3172.16 * * *$ \\
\hline COVID24Apr & $7011.22 * * *$ & -2.22 & -7.24 & $113.25 * * *$ & $611.54^{* * *}$ & $1004.37 * * *$ & $2223.42 * * *$ & $3068.03 * * *$ \\
\hline CoviD1May & $3368.75 * * *$ & -8.14 & -7.95 & $58.70 *$ & $252.46 * * *$ & $411.64 * * *$ & $971.24 * * *$ & $1689.87 * * *$ \\
\hline COVID8May & $-1922.87 * * *$ & $-28.09 * *$ & 1.03 & $-53.57^{*}$ & $-273.94 * * *$ & $-253.83 * * *$ & $-542.30 * * *$ & $-772.74 * * *$ \\
\hline LockD3Week & $4623.09 * * *$ & 4.57 & 1.97 & 12.99 & $444.99 * * *$ & $503.63 * * *$ & $1380.07 * * *$ & $2266.42 * * *$ \\
\hline Constant & $-9790.93 * * *$ & 31.74 & $93.23 * *$ & $-363.26 * *$ & $-2662.46 * * *$ & $-3376.78 * * *$ & -582.99 & $-3188.42 * * *$ \\
\hline Log-likelihood & -21612.63 & -1938.70 & -1593.00 & -3081.15 & -4473.52 & -5167.17 & -7841.34 & -13191.98 \\
\hline
\end{tabular}

Note: ${ }^{*}, *$ and ${ }^{* * *}$ denote significant at the $5 \%, 1 \%$ and $0.1 \%$ levels, respectively. 
Table A5: Total, male and female LockD IdentV2 models with the COVID3Week dummy variable and 5 weekly lockdown identifier variables

\begin{tabular}{|c|c|c|c|}
\hline Variable & Total Deaths & Male Deaths & Female Deaths \\
\hline FTSE All & $-0.463 * * *$ & $-0.203 * * *$ & $-0.256 * * *$ \\
\hline Temp & $-245.58 * * *$ & $-96.21 * * *$ & $-150.00 * * *$ \\
\hline Temp ${ }^{2}$ & $4.18 * * *$ & $1.43 * * *$ & $2.79 * * *$ \\
\hline Winter & $389.46 * * *$ & $159.74 * * *$ & $230.30 * * *$ \\
\hline Summer & $47.27^{* *}$ & 9.76 & $36.77 * *$ \\
\hline Share90+ & 207.13 & $-792.09 * * *$ & $979.50 * * *$ \\
\hline PopDen & $70.07 * * *$ & $47.20 * * *$ & $22.88 * * *$ \\
\hline COVID3Week & $1864.45^{* * *}$ & $1225.88 * * *$ & $640.55^{* * *}$ \\
\hline LockD17Apr & $9709.01 * * *$ & $4869.80 * * *$ & $4844.60 * * *$ \\
\hline LockD24Apr & $9382.67 * * *$ & $4664.92 * * *$ & $4722.86 * * *$ \\
\hline LockD1May & $5751.79 * * *$ & $2469.55 * * *$ & $3286.51 * * *$ \\
\hline LockD8May & $472.81 * * *$ & $-171.00 *$ & $647.90 * * *$ \\
\hline LockD15May & $2415.56 * * *$ & $857.72 * * *$ & $1544.66 * * *$ \\
\hline Constant & $-13599.07 * * *$ & $-11036.37 * * *$ & $-2564.74 * * *$ \\
\hline Log-likelihood & -24362.74 & -12857.25 & -14677.09 \\
\hline
\end{tabular}

Note: $*, * *$ and $* * *$ denote significant at the $5 \%, 1 \%$ and $0.1 \%$ levels, respectively. 
Table A6: Age category LockD IdentV2 models with the COVID3Week dummy variable and 5 weekly lockdown identifier variables

\begin{tabular}{|c|c|c|c|c|c|c|c|c|}
\hline \multirow[b]{2}{*}{ Variable } & \multirow{2}{*}{ Total Deaths } & \multicolumn{7}{|c|}{ Deaths Age Category } \\
\hline & & $<1$ & $1-14$ & $15-44$ & $45-64$ & $65-74$ & $75-84$ & $85+$ \\
\hline FTSE All & $-0.463 * * *$ & -0.001 & 0.001 & 0.007 & $-0.053 * * *$ & $-0.067 * * *$ & $-0.154 * * *$ & $-0.202 * * *$ \\
\hline Temp & $-245.58 * * *$ & $0.96 *$ & -0.44 & -1 & $-19.39 * * *$ & $-21.64 * * *$ & $-72.22 * * *$ & $-131.27 * * *$ \\
\hline Temp ${ }^{2}$ & $4.18^{* * *}$ & -0.04 & 0.01 & -0.04 & $0.44 * * *$ & $0.24^{*}$ & $1.23^{* * *}$ & $2.34 * * *$ \\
\hline Winter & $389.46 * * *$ & 1.08 & -0.02 & -3.24 & $18.30 * * *$ & $53.36 * * *$ & $95.39 * * *$ & $225.30 * * *$ \\
\hline Summer & $47.27^{* *}$ & -0.57 & -0.93 & 2.8 & 0.25 & 9.15 & 18.13 & 17.06 \\
\hline Share90+ & 207.13 & $-72.54 * * *$ & 5.28 & $-385.33 * * *$ & $-1191.86^{* * *}$ & $-372.28 * * *$ & -96.62 & $2264.91 * * *$ \\
\hline PopDen & $70.07 * * *$ & 0.2 & -0.21 & $2.60 * * *$ & $14.74 * * *$ & $16.54 * * *$ & $15.34 * * *$ & $21.59 * * *$ \\
\hline COVID3Week & $1864.45^{* * *}$ & -1.06 & -0.78 & $14.74^{*}$ & $219.35 * * *$ & $288.99 * * *$ & $662.75 * * *$ & $681.22 * * *$ \\
\hline LockD17Apr & $9709.01 * * *$ & 1.08 & -1.31 & $60.09 * *$ & $804.26 * * *$ & $1297.56 * * *$ & $2946.95 * * *$ & $4594.92 * * *$ \\
\hline LockD24Apr & $9382.67 * * *$ & 4.14 & -4.35 & $110.69 * * *$ & $796.41 * * *$ & $1159.59 * * *$ & $2812.15^{* * *}$ & $4498.95^{* * *}$ \\
\hline LockD1May & $5751.79 * * *$ & -1.81 & -5.08 & $56.09 * *$ & $438.44 * * *$ & $568.46 * * *$ & $1563.98 * * *$ & $3125.69 * * *$ \\
\hline LockD8May & $472.81 * * *$ & $-21.78 * * *$ & 3.9 & $-56.15 * * *$ & $-86.63^{*}$ & $-95.07^{*}$ & 54.64 & $668.10 * * *$ \\
\hline LockD15May & $2415.56 * * *$ & 6.27 & 2.86 & -2.54 & $189.42 * * *$ & $161.82 * *$ & $603.54 * * *$ & $1448.73 * * *$ \\
\hline Constant & $-13599.07^{* * *}$ & 38.91 & $94.12 * *$ & $-373.06^{* *}$ & $-3057.04^{* * *}$ & $-3963.00 * * *$ & $-1837.73 * * *$ & $-4721.10 * * *$ \\
\hline Log-likelihood & -24362.74 & -1941.41 & -1596.15 & -3086.21 & -4725.66 & -5570.74 & -8868.47 & -14341.78 \\
\hline
\end{tabular}

